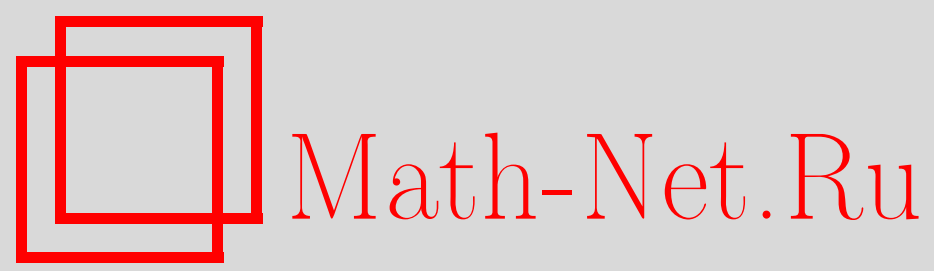

В. А. Ковалёв, Ю. Н. Радаев, Оптимальные системы одномерных подалгебр алгебры симметрий трёхмерных уравнений математической теории пластичности, Вестн. Сам. гос. техн. ун-та. Сер. Физ.-мат. науки, 2011, выпуск 1(), 196-220

DOI: https://doi.org/10.14498/vsgtu860

Использование Общероссийского математического портала Math-Net.Ru подразумевает, что вы прочитали и согласны с пользовательским соглашением http://www.mathnet.ru/rus/agreement

Параметры загрузки:

IP : 54.174 .149 .18

26 апреля 2023 г., 13:11:56 
УДК 539.3

\title{
ОПТИМАЛЬНЫЕ СИСТЕМЫ ОДНОМЕРНЫХ ПОДАЛГЕБР АЛГЕБРЫ СИММЕТРИЙ ТРЁХМЕРНЫХ УРАВНЕНИЙ МАТЕМАТИЧЕСКОЙ ТЕОРИИ ПЛАСТИЧНОСТИ
}

\author{
В. А. Ковалёв ${ }^{1}$ Ю. Н. Радаев ${ }^{2}$ \\ 1 Московский городской университет управления Правительства Москвы, \\ 107045, Москва, ул. Сретенка, 28. \\ 2 Институт проблем механики им. А. Ю. Ишлинского РАН, \\ 119526, Москва, просп. Вернадского, 101. \\ E-mail: vlad_koval@mail.ru; y.radayev@gmail.com
}

Рассматривается естественная конечномерная (размерности 12) подалгебра алгебры симметрий, соответствующей группе симметрий, предложенной в 1959 г. Д. Д. Ивлевым трёхмерных гиперболических уравнений пространственной задачи теории идеальной пластичности для состояний, отвечающих ребру призмы Кулона-Треска, сбормулированных в изостатической системе координат. Приводится алгоритм построения оптимальной системы одномерных подалгебр указанной естественной конечномерной подалгебры алгебры симметрий, насчитывающей один трёхпараметрический элемент, 12 двухпараметрических, 66 однопараметрических элементов и 108 индивидуальных элементов (всего 187 элементов). Ранее было показано, что алгебра симметрий уравнений плоской задачи имеет размерность 7; оптимальная система одномерных подалгебр состоит из 1 двухпараметрического, 11 однопараметрических и 20 индивидуальных инфинитезималъных генераторов (всего 32 элемента). Алгебра симметрий уравнений осесимметричной задачи имеет размерность 5; оптимальная система одномерных подалгебр состоит из 1 однопараметрического и 22 индивидуальных инфинитезималъных генераторов (всего 23 элемента).

Ключевые слова: теория пластичности, изостатические координаты, группа симметрий, алгебра симметрий, подалгебра, оптималъная система, алгоритм.

1. Пространственная задача теории идеальной пластичности (см. [1-5]) при условии пластичности Мизеса и ассоциированным с ним законом течения Леви-Мизеса является статически неопределимой, и, кроме того, уравнения пространственной задачи не гиперболичны. Так, система уравнений пространственной задачи теории идеальной пластичности при условии пластичности Мизеса, вообще говоря, не имеет вещественных характеристических направлений. Точнее говоря, уравнения пространственной задачи либо полностью эллиптичны (т. е. не существует действительных характеристических направлений), либо (если в рассматриваемой точке медианная главная скорость пластической деформации равна нулю) имеется только два двумерных характеристических элемента, совпадающих с площадками максимального касательного напряжения. Все это свидетельствует о том, что в подавляющем большинстве пространственных состояний, описываемых согласно условию пластичности Мизеса и ассоциированному с ним закону течения ЛевиМизеса, действительные характеристики отсутствуют. Как представляется, задача поиска такой математической теории идеальной пластичности, кото-

Владимир Александрович Ковалёв (д.ф.-м.н., профессор), зав. кафедрой, каф. прикладной математики. Юрий Николаевич Радаев (д.ф.-м.н., профессор), ведущий научный сотрудник, лаб. моделирования в механике деформируемого твёрдого тела. 
рая приводила бы в зоне пластического течения к соотношениям гиперболического типа для произвольных пространственных состояний, до настоящего времени сохраняет свою актуальность, поскольку при использовании условий пластичности, отличных от условия пластичности Кулона-Треска, для огромного большинства пространственных состояний уравнения теории пластичности не имеют вещественных характеристических направлений. Все это не оставляет шансов обобщить методы интегрирования существенно нелинейных двумерных уравнений математической теории пластичности, развитые для плоской задачи, соотношения которой формально статически определимы и гиперболичны, что в конце концов и позволяет построить геометрическую теорию полей скольжения, по существу, адекватно представляющую сдвиговый механизм пластического течения. Принципиально иная ситуация наблюдается в пространственной задаче при использовании критерия текучести Кулона-Треска. Здесь уравнения математической теории пластичности в ряде важных случаев становятся гиперболическими. Это прежде всего относится к состояниям, соответствующим ребру призмы Кулона-Треска. $\mathrm{K}$ настоящему времени уже стало ясно, что предельные состояния твёрдых тел также должны описываться статически определимыми уравнениями гиперболического аналитического типа [6].

Для ребра призмы Кулона-Треска, определяемого условием «полной пластичности» Хаара-Кармана $\sigma_{1}=\sigma_{2}=\sigma_{3} \pm 2 k\left(\sigma_{1}, \sigma_{2}, \sigma_{3}\right.$ - главные нормальные напряжения; $k$ - предел текучести при сдвиге), уравнения равновесия, полученные впервые Д. Д. Ивлевым в 1959 г. [7], можно представить в форме одного векторного уравнения (см. статьи $[8,9]$, а также монографию [10])

$$
\operatorname{grad} \sigma_{3} \mp 2 k \operatorname{div}(\boldsymbol{n} \otimes \boldsymbol{n})=\mathbf{0},
$$

где $\boldsymbol{n}$ - единичное векторное поле, имеющее направление главной оси тензора напряжений, соответствующей наибольшему (наименьшему) собственному значению $\sigma_{3}$ тензора напряжений. Уравнение (1) принадлежит к гиперболическому аналитическому типу, нормали к характеристическим поверхностям образуют круговой конус с углом полураствора $\pi / 4$ и осью, направленной вдоль вектора $\boldsymbol{n}$; направления, ортогональные вектору $\boldsymbol{n}$, тоже указывают ориентацию характеристических элементов.

Векторное уравнение (1) может иметь решения с нетривиальной геометрией линий поля $\boldsymbol{n}$ только если указанное поле является расслоенным [10]. Критерием расслоенности векторного поля $\boldsymbol{n}$, в некоторой пространственной области выступает уравнение Якоби

$$
\boldsymbol{n} \cdot \operatorname{rot} \boldsymbol{n}=0 .
$$

Условие расслоенности поля $\boldsymbol{n}$ позволяет ввести 2/3-ортогональные криволинейные координаты $\omega^{\alpha}(\alpha=1,2,3)$, определяемые по векторному полю $\boldsymbol{n}$ так, что координатные поверхности $\omega^{3}=$ const являются слоями поля $\boldsymbol{n}$. Координатные линии на слое векторного поля $\boldsymbol{n}$ могут пересекаться под произвольным углом; третья координатная линия ортогональна слою и ортогональна первым двум координатным линиям. 
Для нахождения соответствующей замены координат $\left(x_{j}\right.$ - пространственные декартовы координаты)

$$
x_{j}=f_{j}\left(\omega^{1}, \omega^{2}, \omega^{3}\right) \quad(j=1,2,3)
$$

может быть получена следующая нелинейная система дифференциальных уравнений в частных производных:

$$
\left\{\begin{array}{l}
\frac{\partial f_{1}}{\partial \omega^{1}} \frac{\partial f_{1}}{\partial \omega^{3}}+\frac{\partial f_{2}}{\partial \omega^{1}} \frac{\partial f_{2}}{\partial \omega^{3}}+\frac{\partial f_{3}}{\partial \omega^{1}} \frac{\partial f_{3}}{\partial \omega^{3}}=0 \\
\frac{\partial f_{1}}{\partial \omega^{2}} \frac{\partial f_{1}}{\partial \omega^{3}}+\frac{\partial f_{2}}{\partial \omega^{2}} \frac{\partial f_{2}}{\partial \omega^{3}}+\frac{\partial f_{3}}{\partial \omega^{2}} \frac{\partial f_{3}}{\partial \omega^{3}}=0 \\
\left(\frac{\partial f_{2}}{\partial \omega^{1}} \frac{\partial f_{3}}{\partial \omega^{2}}-\frac{\partial f_{3}}{\partial \omega^{1}} \frac{\partial f_{2}}{\partial \omega^{2}}\right) \\
\frac{\partial f_{1}}{\partial \omega^{3}}+\left(\frac{\partial f_{3}}{\partial \omega^{1}} \frac{\partial f_{1}}{\partial \omega^{2}}-\frac{\partial f_{1}}{\partial \omega^{1}} \frac{\partial f_{3}}{\partial \omega^{2}}\right) \frac{\partial f_{2}}{\partial \omega^{3}}+ \\
+\left(\frac{\partial f_{1}}{\partial \omega^{1}} \frac{\partial f_{2}}{\partial \omega^{2}}-\frac{\partial f_{2}}{\partial \omega^{1}} \frac{\partial f_{1}}{\partial \omega^{2}}\right) \frac{\partial f_{3}}{\partial \omega^{3}}-1=0
\end{array}\right.
$$

2. Методы группового анализа применительно к системам дифференциальных уравнений в частных производных изложены в классической монографии [11]. Мы будем придерживаться терминологии и обозначений, принятых именно в этой книге. Мы рекомендуем также монографии [12-14] для ознакомления с основами группового анализа дифференциальных уравнений.

Применяя методы группового анализа дифференциальных уравнений, можно вычислить инфинитезимальный генератор $\varsigma \cdot \partial$ группы симметрий системы дифференциальных уравнений (3), зависящий от 12 произвольных постоянных:

$$
\begin{aligned}
& (\varsigma \cdot \partial)=C_{1}\left(\varsigma_{1} \cdot \partial\right)+C_{2}\left(\varsigma_{2} \cdot \partial\right)+C_{3}\left(\varsigma_{3} \cdot \partial\right)+B_{1}\left(\varsigma_{4} \cdot \partial\right)+B_{2}\left(\varsigma_{5} \cdot \partial\right)+B_{3}\left(\varsigma_{6} \cdot \partial\right)+ \\
& +A_{1}\left(\varsigma_{7} \cdot \partial\right)+A_{2}\left(\varsigma_{8} \cdot \partial\right)+A_{3}\left(\varsigma_{9} \cdot \partial\right)+C_{10}\left(\varsigma_{10} \cdot \partial\right)+C_{11}\left(\varsigma_{11} \cdot \partial\right)+C_{12}\left(\varsigma_{12} \cdot \partial\right),
\end{aligned}
$$

где базисные инфинитезимальные генераторы определены согласно

$$
\begin{array}{lll}
\left(\varsigma_{1} \cdot \partial\right)=3 \omega^{3} \frac{\partial}{\partial \omega^{3}}+f_{1} \frac{\partial}{\partial f_{1}}+f_{2} \frac{\partial}{\partial f_{2}}+f_{3} \frac{\partial}{\partial f_{3}}, & \left(\varsigma_{8} \cdot \partial\right)=f_{1} \frac{\partial}{\partial f_{3}}-f_{3} \frac{\partial}{\partial f_{1}}, \\
\left(\varsigma_{2} \cdot \partial\right)=\omega^{3} \frac{\partial}{\partial \omega^{3}}-\frac{\omega^{1}}{2} \frac{\partial}{\partial \omega^{1}}-\frac{\omega^{2}}{2} \frac{\partial}{\partial \omega^{2}}, & \left(\varsigma_{9} \cdot \partial\right)=f_{2} \frac{\partial}{\partial f_{1}}-f_{1} \frac{\partial}{\partial f_{2}}, \\
\left(\varsigma_{3} \cdot \partial\right)=\frac{\partial}{\partial \omega^{3}}, \quad\left(\varsigma_{4} \cdot \partial\right)=\frac{\partial}{\partial f_{1}}, & \left(\varsigma_{10} \cdot \partial\right)=\frac{\partial}{\partial \omega^{1}}, \\
\left(\varsigma_{5} \cdot \partial\right)=\frac{\partial}{\partial f_{2}}, \quad\left(\varsigma_{6} \cdot \partial\right)=\frac{\partial}{\partial f_{3}}, & \left(\varsigma_{11} \cdot \partial\right)=\frac{\partial}{\partial \omega^{2}}, \\
\left(\varsigma_{7} \cdot \partial\right)=f_{3} \frac{\partial}{\partial f_{2}}-f_{2} \frac{\partial}{\partial f_{3}}, & \left(\varsigma_{12} \cdot \partial\right)=\omega^{1} \frac{\partial}{\partial \omega^{1}}-\omega^{2} \frac{\partial}{\partial \omega^{2}} .
\end{array}
$$

В приведённом выше списке инфинитезимальные операторы $\left(\varsigma_{4} \cdot \partial\right),\left(\varsigma_{5} \cdot \partial\right)$, $\left(\varsigma_{6} \cdot \partial\right)$ соответствуют группам переносов вдоль декартовых осей $x_{1}, x_{2}, x_{3}$; инфинитезимальные операторы $\left(\varsigma_{7} \cdot \partial\right),\left(\varsigma_{8} \cdot \partial\right),\left(\varsigma_{9} \cdot \partial\right)$ соответствуют группам 
поворотов вокруг координатных осей $x_{1}, x_{2}, x_{3}$; инфинитезимальные операторы $\left(\varsigma_{3} \cdot \partial\right),\left(\varsigma_{10} \cdot \partial\right),\left(\varsigma_{11} \cdot \partial\right)$ соответствуют группам трансляций изостатических координат $\omega^{1}, \omega^{2}, \omega^{3}$; инфинитезимальный оператор $\left(\varsigma_{12} \cdot \partial\right)$ определяет группу, инфинитезимально сохраняющую площадь двумерного плоского элемента $d \omega^{1} d \omega^{2} ;$ инфинитезимальный оператор $\left(\varsigma_{1} \cdot \partial\right)$ соответствует группе совместных растяжений координат $\omega^{3}, x_{1}, x_{2}, x_{3}$ в подходящих пропорциях; инфинитезимальный оператор $\left(\varsigma_{2} \cdot \partial\right)$ соответствует группе совместных растяженийсжатий изостатических координат $\omega^{1}, \omega^{2}, \omega^{3}$ в подходящих пропорциях.

Известно, что алгебра симметрий пространственных уравнений математической теории пластичности (3) бесконечномерна. Можно показать, что инфинитезимальные операторы $\left(\varsigma_{j} \cdot \partial\right)(j=1,2, \ldots, 12)$ линейно независимы. Поэтому можно ввести конечномерное линейное подпространство, представляющее собой линейную оболочку операторов $\left(\varsigma_{j} \cdot \partial\right)$. Двенадцатимерное линейное пространство с базисом из инфинитезимальных операторов (5) наделяется стандартной алгебраической структурой с помощью билинейной операции коммутации операторов (скобка Пуассона операторов). Чтобы доказать, что линейная оболочка операторов (5) образует алгебру Ли, необходимо составить таблицу коммутаторов базисных инфинитезимальных операторов $\left(\varsigma_{j} \cdot \partial\right)(j=1,2, \ldots, 12)$, проверив при этом, что коммутаторы $\left[\left(\varsigma_{i} \cdot \partial\right),\left(\varsigma_{j} \cdot \partial\right)\right]$ снова можно разложить по базису $\left(\varsigma_{k} \cdot \partial\right)$ :

$$
\left[\left(\varsigma_{i} \cdot \partial\right),\left(\varsigma_{j} \cdot \partial\right)\right]=C_{\cdot i j}^{k \cdot *}\left(\varsigma_{k} \cdot \partial\right)
$$

Символы $C_{. i j}^{k \cdot \bullet}$ в разложении (6) являются структурными константами алгебры Ли в базисе $\left(\varsigma_{j} \cdot \partial\right)(j=1,2, \ldots, 12)$.

Таблица коммутаторов, приведенная ниже, показывает, что инфинитезимальные операторы (5) действительно определяют конечномерную подалгебру $L^{12}$ алгебры симметрий системы дифференциальных уравнений в частных производных (3). Таблица составлена так, чтобы на пересечении строки с номером $i$ и столбца с номером $j$ находился коммутатор $\left[\left(\varsigma_{i} \cdot \partial\right),\left(\varsigma_{j} \cdot \partial\right)\right]$.

Структурные константы $C_{\text {.ij }}^{k \cdot *}$ рассматриваемой конечномерной алгебры Ли $L^{12}$ без труда определяются на основании приведенной таблицы коммутаторов. Здесь мы приводим ненулевые структурные константы алгебры Ли $L^{12}$ в базисе $\left(\varsigma_{j} \cdot \partial\right)(j=1,2, \ldots, 12)$ :

$$
\begin{aligned}
& C_{\cdot 13}^{3 \cdot \cdot}=-3, \quad C_{\cdot 14}^{4 \cdot *}=-1, \quad C_{\cdot 15}^{5 \cdot \cdot}=-1, \quad C_{\cdot 16}^{6 \cdot *}=-1 ; \\
& C_{\cdot 23}^{3 \cdot *}=-1, \quad C_{\cdot 2 \underline{10}}^{10 \cdot *}=1 / 2, \quad C_{\cdot 2 \underline{11}}^{11 \cdot *}=1 / 2 \text {; } \\
& C_{31}^{3 . *}=3, \quad C_{.32}^{3 . \cdot}=1 ; \\
& C_{.41}^{4 . *}=1, \quad C_{.48}^{6 . *}=1, \quad C_{.49}^{5 . \cdot}=-1 ; \\
& C_{.51}^{5 . .}=1, \quad C_{.57}^{6 . .}=-1, \quad C_{.59}^{4 . \cdot}=1 ; \\
& C_{.61}^{6 \cdot .}=1, \quad C_{.67}^{5 . *}=1, \quad C_{.68}^{4 . *}=-1 ; \\
& C_{.75}^{6 . *}=1, \quad C_{.76}^{5 . *}=-1, \quad C_{.78}^{9 . *}=1, \quad C_{.79}^{8 . *}=-1 \text {; } \\
& C_{.84}^{6 . *}=-1, \quad C_{.86}^{4 . \cdot}=1, \quad C_{.87}^{9 . \cdot}=-1, \quad C_{.89}^{7 . \cdot}=1 ; \\
& C_{.94}^{5 \cdot *}=1, \quad C_{.95}^{4 \cdot .}=-1, \quad C_{.97}^{8 \cdot \cdot}=1, \quad C_{.98}^{7 \cdot *}=-1 ; \\
& C \frac{10 \cdot \cdot}{\cdot 102}=-1 / 2, \quad C \cdot \frac{10 \cdot \cdot}{1012}=1 \text {; }
\end{aligned}
$$

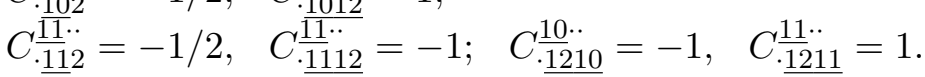


Заметим, что натуральные числа, записываемые двумя цифрами, подчеркнуты снизу для того, чтобы отличать их от расположенных друг за другом натуральных чисел, представляемых одной цифрой.

Далее можно указать однопараметрические группы автоморфизмов рассматриваемой алгебры Ли $L^{12}$, порождаемые базисными векторами $\varsigma_{j}(j=$ $=1,2, \ldots, 12)$. Для каждого базисного вектора $\varsigma_{j}(j=1,2, \ldots, 12)$ имеем соответствующую однопараметрическую группу внутренних автоморфизмов, действующую на коэффициенты $C_{1}, C_{2}, C_{3}, A_{1}, A_{2}, A_{3}, B_{1}, B_{2}, B_{3}, C_{10}, C_{11}$, $C_{12}$; они приводятся в приложении $\mathrm{A}$.

3. Построение оптимальной системы одномерных подалгебр алгебры симметрий $L^{12}$ осуществим с помощью «наивного» подхода, состоящего в том, что инфинитезимальный оператор (4) (точнее, коэффициенты $C_{1}, C_{2}, C_{3}$, $\left.A_{1}, A_{2}, A_{3}, B_{1}, B_{2}, B_{3}, C_{10}, C_{11}, C_{12}\right)$ подвергается различным преобразованиям из приложения А так, чтобы «упростить» его настолько, насколько это представляется возможным (в частности, стремясь привести к нулевому значению как можно больше из указанных двенадцати постоянных). Далее мы выбираем из каждого класса инфинитезимальных операторов, переводящихся друг в друга автоморфизмами A1)-A12), по одному простейшему представителю и формируем оптимальную систему одномерных подалгебр алгебры непрерывных симметрий системы дифференциальных уравнений в частных производных (3).

При поиске указанных простейших представителей кроме однопараметрических групп автоморфизмов будем применять также преобразование, заключающееся в умножении простейшего инфинитезимального оператора на некоторую постоянную (так называемое преобразование умножения).

Рассмотрим $A_{i}$ и $B_{i}$ как компоненты векторов $\boldsymbol{A}$ и $\boldsymbol{B}$ в трёхмерном пространстве $x_{1}, x_{2}, x_{3}$. Тогда автоморфизмы A7)-А9) представляют собой повороты указанных векторов как жесткого целого на различные углы $\tau$ вокруг декартовых осей $x_{1}, x_{2}, x_{3}$.

Если вектор $\boldsymbol{A}$ ненулевой (т. е. хотя бы одна из его компонент $A_{i}$ не равна нулю), то такими поворотами можно перевести вектор $\boldsymbol{A}$ в положение, когда он будет коллинеарен оси $x_{1}$. Ясно, что тогда $A_{2}=A_{3}=0, A_{1} \neq 0$. При этом, если вектор $\boldsymbol{B}$ не равен нулю (т. е. хотя бы одна из его компонент $B_{i}$ отлична от нуля), то поворотом вокруг оси $x_{1}$ вектор $\boldsymbol{B}$ можно преобразовать так, чтобы его проекция на ось $x_{3}$ (т. е. компонента $B_{3}$ ) была равна нулю $\left(B_{3}=0\right)$. Применяя последовательно автоморфизмы A5), A6) при значениях $\tau$, равных соответственно $B_{2} C_{1} /\left(C_{1}^{2}+A_{1}^{2}\right)$ и $B_{2} A_{1} /\left(C_{1}^{2}+A_{1}^{2}\right)$, можно добиться того, чтобы компонента $B_{2}$ стала нулевой, не изменяя при этом нулевого значения компоненты $B_{3}$.

Если вектор $\boldsymbol{A}$ равен нулю (т. е. $A_{1}=A_{2}=A_{3}=0$ ), то поворотами А7)A9) вектор $\boldsymbol{B}$ заведомо может быть переведён в такое положение, когда он будет коллинеарен оси $x_{1}$, и поэтому снова получаем $B_{2}=B_{3}=0$.

Таким образом, при любых обстоятельствах можно добиться того, чтобы выполнялись равенства $A_{2}=A_{3}=0$ и $B_{2}=B_{3}=0$. Поскольку декартовы оси $x_{j}(j=1,2,3)$ равноправны, то вместо $A_{2}=A_{3}=0$ и $B_{2}=B_{3}=0$ можно считать выполненными равенства $A_{1}=A_{3}=0$ и $B_{1}=B_{3}=0$ или $A_{1}=A_{2}=0$ и $B_{1}=B_{2}=0$. 


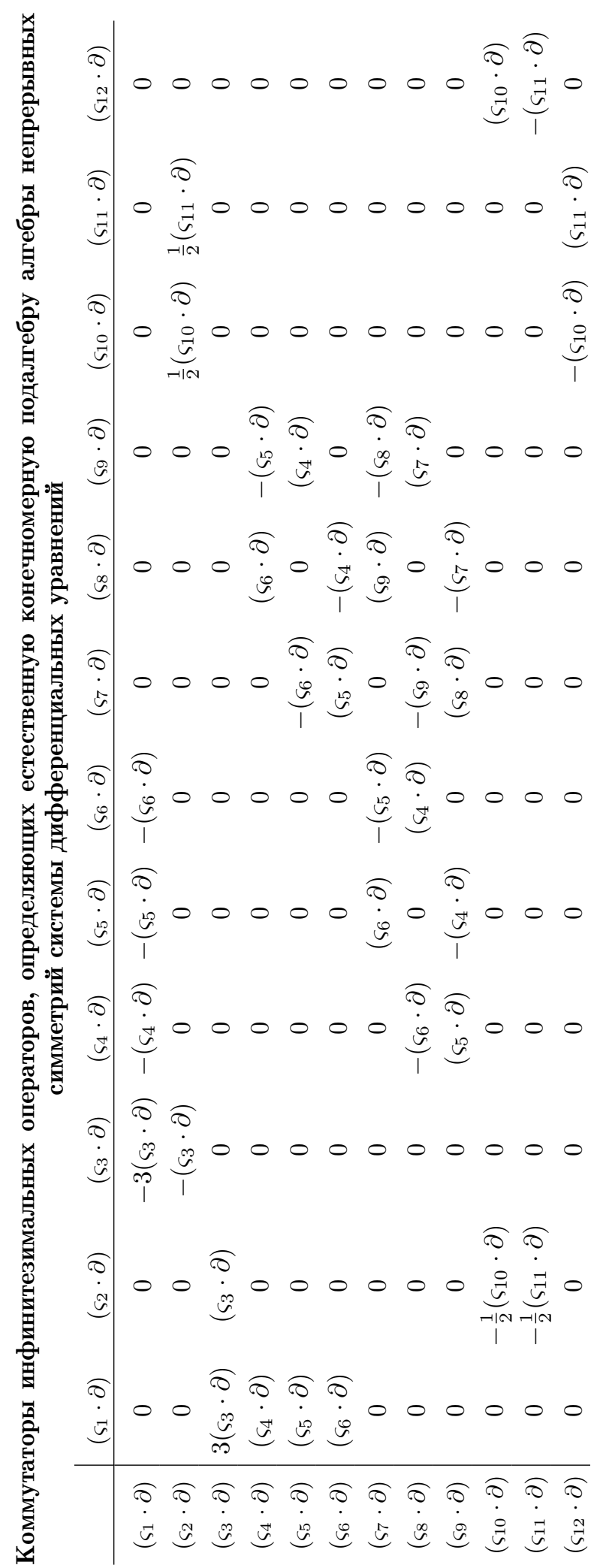


Следует отметить, что если $C_{1} \neq 0$, то применяя автоморфизм $\left.\mathrm{A} 4\right)$ при $\tau=B_{1} / C_{1}$, удаётся привести к нулевому значению компоненту $B_{1}$ вектора $\boldsymbol{B}$.

Дальнейшие рассуждения удобнее всего разбить на четыре этапа, характеризующихся выполнением перечисленных ниже условий: I) $C_{2} \pm 2 C_{12} \neq 0$; II) $C_{2}-2 C_{12}=0, C_{2}+2 C_{12} \neq 0$; III) $C_{2}+2 C_{12}=0, C_{2}-2 C_{12} \neq 0$; IV) $C_{2}=0$, $2 C_{12}=0$.

ЭТАП І. На протяжении всего первого этапа будем считать, что выполняется условие $C_{2} \pm 2 C_{12} \neq 0$. Если $C_{2}, C_{12}$ выбираются так, что $C_{2} \pm 2 C_{12} \neq 0$, то, применяя автоморфизмы $\left.\mathrm{A} 10\right)$ и $\left.\mathrm{A} 11\right)$ при $\tau$, соответственно равном $2 C_{10} /\left(2 C_{12}-C_{2}\right)$ и $-2 C_{11} /\left(2 C_{12}+C_{2}\right)$, можно привести к нулевому значению $C_{10}$ и $C_{11}$.

I.A. Коэффициенты $C_{1}, C_{2}$ подчиняются неравенству $3 C_{1}+C_{2} \neq 0$. При выполнении неравенства $3 C_{1}+C_{2} \neq 0$, применяя автоморфизм А3) при $\tau=C_{3} /\left(3 C_{1}+C_{2}\right)$, можно привести к нулевому значению $C_{3}$.

I.A.1. Считаем выполненным условие $C_{1} \neq 0$. При условии $C_{1} \neq 0$, как отмечалось выше, удаётся привести к нулевому значению $B_{1}$; применяя затем преобразование умножения, приводим $C_{1}$ к единице и, таким образом, получаем множество простейших представителей вида

$$
\left(\varsigma_{1} \cdot \partial\right)+D_{1}\left(\varsigma_{2} \cdot \partial\right)+D_{2}\left(\varsigma_{7} \cdot \partial\right)+D_{3}\left(\varsigma_{12} \cdot \partial\right),
$$

где $D_{1}, D_{2}, D_{3}$ - произвольные постоянные, подчиненные ограничению $\left|D_{1}\right| \neq 2\left|D_{3}\right|$.

I.A.2. Этот этап рассуждений характеризуется выполнением дополнительного условия $C_{1}=0$. Если $C_{1}=0$, то коэффициент $B_{1}$ привести к нулевому значению не удаётся.

I.A.2-1. Если, кроме того, $C_{2} \neq 0$ и $B_{1} \neq 0$, то, применяя автоморфизм A1) при $\tau$, равном $\ln \left|C_{2} / B_{1}\right|$, добиваемся того, чтобы коэффициенты $C_{2}$ и $B_{1}$ стали равными по абсолютной величине. В результате получаем простейших представителей вида

$$
\left(\varsigma_{2} \cdot \partial\right) \pm\left(\varsigma_{4} \cdot \partial\right)+D_{1}\left(\varsigma_{7} \cdot \partial\right)+D_{2}\left(\varsigma_{12} \cdot \partial\right) .
$$

I.A.2-2. Если полагать $C_{2} \neq 0$ и $B_{1}=0$, то получаем следующих простейших представителей:

$$
\left(\varsigma_{2} \cdot \partial\right)+D_{1}\left(\varsigma_{7} \cdot \partial\right)+D_{2}\left(\varsigma_{12} \cdot \partial\right) .
$$

I.В. Коэффициенты $C_{1}, C_{2}$ подчиняются равенству $3 C_{1}+C_{2}=0$. Если $3 C_{1}+C_{2}=0$, то коэффициент $C_{3}$ сделать нулевым не удаётся.

I.В.1. Считаем выполненным условие $C_{1} \neq 0$. Тогда удаётся привести к нулевому значению коэффициент $B_{1}$.

I.В.1-1. Если, кроме того, $C_{3} \neq 0$, то, применяя автоморфизм А2) при $\tau=$ $=\ln \left|C_{1} / C_{3}\right|$, приводим $C_{1}$ и $C_{3}$ к значениям, равным по модулю; учитывая $3 C_{1}+C_{2}=0$, получаем множество простейших представителей вида

$$
\left(\varsigma_{1} \cdot \partial\right)-3\left(\varsigma_{2} \cdot \partial\right) \pm\left(\varsigma_{3} \cdot \partial\right)+D_{1}\left(\varsigma_{7} \cdot \partial\right)+D_{2}\left(\varsigma_{12} \cdot \partial\right) .
$$


I.В.1-2. Если $C_{3}=0$, то получаем двухпараметрическое семейство простейших представителей:

$$
\left(\varsigma_{1} \cdot \partial\right)-3\left(\varsigma_{2} \cdot \partial\right)+D_{1}\left(\varsigma_{7} \cdot \partial\right)+D_{2}\left(\varsigma_{12} \cdot \partial\right) .
$$

I.В.2. Считаем выполненным условие $C_{1}=0$. Тогда в силу $3 C_{1}+C_{2}=0$ будет выполнено условие $C_{2}=0$. Ясно, что при этом $C_{12} \neq 0$. Если $C_{1}=0$, то коэффициент $B_{1}$ привести к нулевому значению не удаётся. В случае, когда $C_{1}=0$ и $C_{2}=0$, находим, что коэффициенты $C_{3}$ и $B_{1}$ не приводятся к нулевому значению.

I.В. $2-1$. Полагаем, что $C_{3} \neq 0, B_{1} \neq 0, A_{1} \neq 0$. Если $C_{3} \neq 0, B_{1} \neq 0, A_{1} \neq 0$, то, применяя автоморфизм А1) при $\tau=\ln \left|A_{1} / B_{1}\right|$, убеждаемся, что $A_{1}$ и $B_{1}$ приводятся к равным по модулю значениям, и, применяя после этого автоморфизм А2) при $\tau=\ln \left|A_{1} / C_{3}\right|$, приводим к равным абсолютным значениям коэффициенты $A_{1}$ и $C_{3}$. В итоге получаем однопараметрическое семейство простейших представителей:

$$
\left(\varsigma_{3} \cdot \partial\right) \pm\left(\varsigma_{4} \cdot \partial\right) \pm\left(\varsigma_{7} \cdot \partial\right)+D\left(\varsigma_{12} \cdot \partial\right) \quad(D \neq 0) .
$$

Здесь $D$ - произвольная постоянная.

I.В.2-2. Полагаем, что $C_{3} \neq 0, B_{1}=0, A_{1} \neq 0$. Применяя автоморфизм A1), приводим к равным абсолютным значениям коэффициенты $A_{1}$ и $C_{3}$. Получаем простейших представителей

$$
\left(\varsigma_{3} \cdot \partial\right) \pm\left(\varsigma_{7} \cdot \partial\right)+D\left(\varsigma_{12} \cdot \partial\right) \quad(D \neq 0) .
$$

I.В.2-3. Полагаем, что $C_{3}=0, B_{1} \neq 0, A_{1} \neq 0$. Применяя автоморфизм A1), приводим к равным абсолютным значениям коэффициенты $A_{1}$ и $B_{1}$. Получаем простейших представителей

$$
\left(\varsigma_{4} \cdot \partial\right) \pm\left(\varsigma_{7} \cdot \partial\right)+D\left(\varsigma_{12} \cdot \partial\right) \quad(D \neq 0) .
$$

I.B.2-4. Полагаем, что $C_{3} \neq 0, B_{1} \neq 0, A_{1}=0$. Напомним, что $C_{12} \neq 0$. Применяя автоморфизм А1) при $\tau=\ln \left|C_{12} / B_{1}\right|$, что допустимо в силу $C_{12} \neq 0, B_{1} \neq 0$, убеждаемся, что $C_{12}$ и $B_{1}$ приводятся к равным по модулю значениям, и, применяя после этого автоморфизм А2) при $\tau=\ln \left|C_{12} / C_{3}\right|$, что допустимо в силу $C_{12} \neq 0$, $C_{3} \neq 0$, приводим к равным абсолютным значениям величины $C_{12}$ и $C_{3}$. В результате получаем представителей

$$
\left(\varsigma_{3} \cdot \partial\right) \pm\left(\varsigma_{4} \cdot \partial\right) \pm\left(\varsigma_{12} \cdot \partial\right) .
$$

I.В.2-5. Полагаем, что $C_{3} \neq 0, B_{1}=0, A_{1}=0$. Применяя автоморфизм A1), приводим к равным абсолютным значениям коэффициенты $C_{3}$ и $C_{12}$, которые в пределах рассматриваемого случая оба отличны от нуля. Получаем простейших представителей

$$
\left(\varsigma_{3} \cdot \partial\right) \pm\left(\varsigma_{12} \cdot \partial\right) .
$$


I.B.2-6. Полагаем, что $C_{3}=0, B_{1} \neq 0, A_{1}=0$. Применяя автоморфизм A1), приводим к равным абсолютным значениям коэффициенты $B_{1}$ и $C_{12}$, которые в пределах рассматриваемого случая оба отличны от нуля. Получаем простейших представителей

$$
\left(\varsigma_{4} \cdot \partial\right) \pm\left(\varsigma_{12} \cdot \partial\right)
$$

I.B.2-7. Полагаем, что $C_{3}=0, B_{1}=0, A_{1} \neq 0$. Коэффициент $C_{12}$ в пределах рассматриваемого случая отличен от нуля. Получаем однопараметрическое семейство простейших представителей:

$$
\left(\varsigma_{7} \cdot \partial\right)+D\left(\varsigma_{12} \cdot \partial\right) \quad(D \neq 0) .
$$

I.B.2-8. Полагаем, что $C_{3}=0, B_{1}=0, A_{1}=0$. Коэффициент $C_{12}$ в пределах рассматриваемого случая отличен от нуля. Получаем простейшего представителя

$$
\left(\varsigma_{12} \cdot \partial\right)
$$

ЭТАП II. Этот этап характеризуется выполнением условий $C_{2}-2 C_{12}=0$ и $C_{2}+2 C_{12} \neq 0$. Если коэффициенты $C_{2}, C_{12}$ выбираются так, что $C_{2}-2 C_{12}=0$, но $C_{2}+2 C_{12} \neq 0$ (т. е. $C_{2}=2 C_{12} \neq 0$ ), то коэффициент $C_{10}$ не удаётся привести к нулевому значению. Применяя автоморфизм А11) при $\tau=-2 C_{11} /\left(2 C_{12}+C_{2}\right)$, можно привести к нулевому значению коэффициент $C_{11}$.

II.А. Считаем выполненным неравенство $3 C_{1}+C_{2} \neq 0$.

II.A.1. Полагаем, кроме того, что удовлетворяется неравенство $C_{1} \neq 0$. Если $C_{1} \neq 0$ и $3 C_{1}+C_{2} \neq 0$, то, применяя автоморфизмы $\left.\mathrm{A} 4\right)$ и А3) при $\tau$, соответственно равном $B_{1} / C_{1}$ и $C_{3} /\left(3 C_{1}+C_{2}\right)$, можно привести к нулевому значению коэффициенты $B_{1}$ и $C_{3}$.

II.A.1-1. Дополнительно полагаем, что $C_{10} \neq 0$. Поскольку $C_{10} \neq 0$, то, применяя автоморфизм A12) при $\tau=\ln \left|C_{1} / C_{10}\right|$, убеждаемся, что коэффициенты $C_{1}$ и $C_{10}$ приводятся к равным по модулю значениям; применяя затем преобразование умножения, приводим коэффициент $C_{1}$ к значению, равному единице и, таким образом, получаем двухпараметрическое семейство простейших представителей вида

$$
\left(\varsigma_{1} \cdot \partial\right) \pm\left(\varsigma_{10} \cdot \partial\right)+D_{1}\left(\left(\varsigma_{2} \cdot \partial\right)+\frac{1}{2}\left(\varsigma_{12} \cdot \partial\right)\right)+D_{2}\left(\varsigma_{7} \cdot \partial\right) \quad\left(D_{1} \neq 0\right) .
$$

II.А.1-2. Этот случай характеризуется выполнением дополнительного равенства $C_{10}=0$. Находим двухпараметрическое семейство простейших представителей

$$
\left(\varsigma_{1} \cdot \partial\right)+D_{1}\left(\left(\varsigma_{2} \cdot \partial\right)+\frac{1}{2}\left(\varsigma_{12} \cdot \partial\right)\right)+D_{2}\left(\varsigma_{7} \cdot \partial\right) \quad\left(D_{1} \neq 0\right) .
$$

Это семейство дополняет (20) при $D_{3}=D_{1} / 2$. 
II.A.2. Полагаем, кроме того, что выполняется равенство $C_{1}=0$. Если $C_{1}=0$, то коэффициент $B_{1}$ привести к нулевому значению не удаётся.

II.A.2-1. Полагаем, что $C_{10} \neq 0, B_{1} \neq 0, C_{2} \neq 0$. Применяя автоморфизм А12) при $\tau=\ln \left|C_{2} / C_{10}\right|$, убеждаемся, что $C_{2}$ и $C_{10}$ приводятся к равным по модулю значениям. Если $C_{2} \neq 0, B_{1} \neq 0$, то, применяя автоморфизм A1) при $\tau=\ln \left|C_{2} / B_{1}\right|$, добиваемся того, чтобы коэффициенты $C_{2}$ и $B_{1}$ стали равными по абсолютной величине. В результате получаем простейших представителей

$$
\left(\varsigma_{2} \cdot \partial\right) \pm\left(\varsigma_{4} \cdot \partial\right) \pm\left(\varsigma_{10} \cdot \partial\right)+\frac{1}{2}\left(\varsigma_{12} \cdot \partial\right)+D\left(\varsigma_{7} \cdot \partial\right) .
$$

II.А.2-2. Полагаем, что $C_{10} \neq 0, B_{1}=0, C_{2} \neq 0$. Получаем следующих простейших представителей:

$$
\left(\varsigma_{2} \cdot \partial\right) \pm\left(\varsigma_{10} \cdot \partial\right)+\frac{1}{2}\left(\varsigma_{12} \cdot \partial\right)+D\left(\varsigma_{7} \cdot \partial\right) .
$$

II.A.2-3. Полагаем, что $C_{10}=0, B_{1} \neq 0, A_{1} \neq 0, C_{2} \neq 0$. Применяя автоморфизм A1), убеждаемся, что $A_{1}$ и $B_{1}$ приводятся к равным по модулю значениям. Получаем следующих простейших представителей:

$$
2\left(\varsigma_{2} \cdot \partial\right)+D\left(\left(\varsigma_{4} \cdot \partial\right) \pm\left(\varsigma_{7} \cdot \partial\right)\right)+\left(\varsigma_{12} \cdot \partial\right) \quad(D \neq 0)
$$

II.А.2-4. Полагаем, что $C_{10}=0, B_{1} \neq 0, A_{1}=0, C_{2} \neq 0$. Получаем следующих простейших представителей:

$$
2\left(\varsigma_{2} \cdot \partial\right) \pm\left(\varsigma_{4} \cdot \partial\right)+\left(\varsigma_{12} \cdot \partial\right) .
$$

II.А.2-5. Полагаем, что $C_{10}=0, B_{1}=0, A_{1} \neq 0, C_{2} \neq 0$. Получаем следующих простейших представителей:

$$
2\left(\varsigma_{2} \cdot \partial\right)+D\left(\varsigma_{7} \cdot \partial\right)+\left(\varsigma_{12} \cdot \partial\right) \quad(D \neq 0) .
$$

II.А.2-6. Полагаем, что $C_{10}=0, B_{1}=0, A_{1}=0, C_{2} \neq 0$. Получаем простейших представителей

$$
2\left(\varsigma_{2} \cdot \partial\right)+\left(\varsigma_{12} \cdot \partial\right)
$$

которые дополняют (25), (27) при $D=0$.

II.B. Считаем дополнительно выполненным равенство $3 C_{1}+C_{2}=0$. Если $3 C_{1}+C_{2}=0$, то коэффициент $C_{3}$ не удаётся привести к нулевому значению.

II.B.1. Полагаем, кроме того, что удовлетворяется неравенство $C_{1} \neq 0$. Тогда можно привести к нулевому значению коэффициент $B_{1}$.

II.B.1-1. Дополнительно считаем, что $C_{3} \neq 0, C_{10} \neq 0$. Если $C_{3} \neq 0, C_{10} \neq 0$, то, применяя автоморфизмы А2) и А12) при $\tau$, равном соответственно $\ln \left|C_{1} / C_{3}\right|$ и $\ln \left|C_{1} / C_{10}\right|$, приводим $C_{1}, C_{3}$ и $C_{10}$ к значениям, равным по модулю, и, следовательно, получаем множество простейших представителей вида

$$
\left(\varsigma_{1} \cdot \partial\right)-3\left(\varsigma_{2} \cdot \partial\right) \pm\left(\varsigma_{3} \cdot \partial\right) \pm\left(\varsigma_{10} \cdot \partial\right)-\frac{3}{2}\left(\varsigma_{12} \cdot \partial\right)+D\left(\varsigma_{7} \cdot \partial\right) .
$$


II.B.1-2. Дополнительно принимаем, что выполнены условия $C_{3} \neq 0, C_{10}=0$. Получаем множество простейших представителей

$$
\left(\varsigma_{1} \cdot \partial\right)-3\left(\varsigma_{2} \cdot \partial\right) \pm\left(\varsigma_{3} \cdot \partial\right)-\frac{3}{2}\left(\varsigma_{12} \cdot \partial\right)+D\left(\varsigma_{7} \cdot \partial\right) .
$$

II.В.1-3. Дополнительно принимаем, что выполнены условия $C_{3}=0, C_{10} \neq 0$. $\mathrm{C}$ помощью автоморфизма $\mathrm{A} 12)$ коэффициенты $C_{10}$ и $C_{1}$ удаётся привести к значениям, одинаковым по абсолютной величине. Учитывая еще, что $3 C_{1}+C_{2}=0$ и $C_{2}=2 C_{12} \neq 0$, получаем множество простейших представителей

$$
\left(\varsigma_{1} \cdot \partial\right)-3\left(\varsigma_{2} \cdot \partial\right) \pm\left(\varsigma_{10} \cdot \partial\right)-\frac{3}{2}\left(\varsigma_{12} \cdot \partial\right)+D\left(\varsigma_{7} \cdot \partial\right) .
$$

II.B.1-4. Дополнительно принимаем, что выполнены условия $C_{3}=0, C_{10}=0$. Получаем множество простейших представителей

$$
-\frac{2}{3}\left(\varsigma_{1} \cdot \partial\right)+2\left(\varsigma_{2} \cdot \partial\right)+D\left(\varsigma_{7} \cdot \partial\right)+\left(\varsigma_{12} \cdot \partial\right) .
$$

II.В.2. Случаи типа II.В.2, когда выполняется равенство $C_{1}=0$, невозможны в рамках предположений II.В.

ЭтАП III. Этот этап характеризуется выполнением условий $C_{2}+2 C_{12}=0$ и $C_{2}-2 C_{12} \neq 0$. Если $C_{2}, C_{12}$ выбираются так, что $C_{2}+2 C_{12}=0$, но $C_{2}-2 C_{12} \neq 0$ (т. е. $C_{2}=-2 C_{12} \neq 0$ ), то коэффициент $C_{11}$ автоморфизмами A1)-A12) не удаётся привести к нулевому значению. Применяя автоморфизм А10) при $\tau=2 C_{10} /\left(2 C_{12}-C_{2}\right)$, можно привести к нулевому значению коэффициент $C_{10}$.

III.A. Считаем выполненным неравенство $3 C_{1}+C_{2} \neq 0$.

III.A.1. Полагаем, кроме того, что удовлетворяется неравенство $C_{1} \neq 0$. Если $C_{1} \neq 0$ и $3 C_{1}+C_{2} \neq 0$, то, применяя автоморфизмы $\left.\mathrm{A} 4\right)$ и $\mathrm{A} 3)$ при $\tau$, соответственно равном $B_{1} / C_{1}$ и $C_{3} /\left(3 C_{1}+C_{2}\right)$, можно привести к нулевому значению $B_{1}$ и $C_{3}$.

III.A.1-1. Дополнительно полагаем, что $C_{11} \neq 0$. Так как $C_{11} \neq 0$, то, применяя автоморфизм A12) при $\tau=-\ln \left|C_{1} / C_{11}\right|$, убеждаемся, что $C_{1}$ и $C_{11}$ приводятся к равным по модулю значениям; применяя затем преобразование умножения, приводим $C_{1}$ к единице и, таким образом, получаем множество простейших представителей вида

$$
\left(\varsigma_{1} \cdot \partial\right) \pm\left(\varsigma_{11} \cdot \partial\right)+D_{1}\left(\left(\varsigma_{2} \cdot \partial\right)-\frac{1}{2}\left(\varsigma_{12} \cdot \partial\right)\right)+D_{2}\left(\varsigma_{7} \cdot \partial\right) \quad\left(D_{1} \neq 0\right) .
$$

III.А.1-2. Этот случай характеризуется выполнением дополнительного равенства $C_{11}=0$. Находим двухпараметрическое семейство простейших представителей:

$$
\left(\varsigma_{1} \cdot \partial\right)+D_{1}\left(\left(\varsigma_{2} \cdot \partial\right)-\frac{1}{2}\left(\varsigma_{12} \cdot \partial\right)\right)+D_{2}\left(\varsigma_{7} \cdot \partial\right) \quad\left(D_{1} \neq 0\right) .
$$

Это семейство дополняет (8) при $D_{3}=-D_{1} / 2$. 
III.А.2. Полагаем, кроме того, что выполняется равенство $C_{1}=0$. Если $C_{1}=0$, то коэффициент $B_{1}$ привести к нулевому значению не удаётся.

III.A.2-1. Полагаем, что $C_{11} \neq 0, B_{1} \neq 0, C_{2} \neq 0$. Применяя автоморфизм А12) при $\tau=-\ln \left|C_{2} / C_{11}\right|$, убеждаемся, что $C_{2}$ и $C_{11}$ приводятся к равным по модулю значениям. Если $B_{1} \neq 0$, то, применяя автоморфизм A1) при $\tau=\ln \left|C_{2} / B_{1}\right|$, добиваемся того, чтобы $C_{2}$ и $B_{1}$ стали равными по абсолютной величине. В результате получаем простейших представителей вида

$$
\left(\varsigma_{2} \cdot \partial\right) \pm\left(\varsigma_{4} \cdot \partial\right) \pm\left(\varsigma_{11} \cdot \partial\right)-\frac{1}{2}\left(\varsigma_{12} \cdot \partial\right)+D\left(\varsigma_{7} \cdot \partial\right) .
$$

III.А.2-2. Полагаем, что $C_{11} \neq 0, B_{1}=0, C_{2} \neq 0$. Получаем следующих простейших представителей:

$$
\left(\varsigma_{2} \cdot \partial\right) \pm\left(\varsigma_{11} \cdot \partial\right)-\frac{1}{2}\left(\varsigma_{12} \cdot \partial\right)+D\left(\varsigma_{7} \cdot \partial\right) .
$$

III.А.2-3. Полагаем, что $C_{11}=0, B_{1} \neq 0, A_{1} \neq 0, C_{2} \neq 0$. Применяя автоморфизм A1), убеждаемся, что $A_{1}$ и $B_{1}$ приводятся к равным по модулю значениям. Получаем следующих простейших представителей:

$$
-2\left(\varsigma_{2} \cdot \partial\right)+D\left(\left(\varsigma_{4} \cdot \partial\right) \pm\left(\varsigma_{7} \cdot \partial\right)\right)+\left(\varsigma_{12} \cdot \partial\right) \quad(D \neq 0) .
$$

III.А.2-4. Полагаем, что $C_{11}=0, B_{1} \neq 0, A_{1}=0, C_{2} \neq 0$. Получаем следующих простейших представителей:

$$
-2\left(\varsigma_{2} \cdot \partial\right) \pm\left(\varsigma_{4} \cdot \partial\right)+\left(\varsigma_{12} \cdot \partial\right)
$$

III.А.2-5. Полагаем, что $C_{11}=0, B_{1}=0, A_{1} \neq 0, C_{2} \neq 0$. Получаем следующих простейших представителей:

$$
-2\left(\varsigma_{2} \cdot \partial\right)+D\left(\varsigma_{7} \cdot \partial\right)+\left(\varsigma_{12} \cdot \partial\right) \quad(D \neq 0) .
$$

III.А.2-6. Полагаем, что $C_{11}=0, B_{1}=0, A_{1}=0, C_{2} \neq 0$. Получаем следующих простейших представителей:

$$
-2\left(\varsigma_{2} \cdot \partial\right)+\left(\varsigma_{12} \cdot \partial\right)
$$

которые дополняют (37), (39) при $D=0$.

III.В. Считаем дополнительно выполненным равенство $3 C_{1}+C_{2}=0$. Если $3 C_{1}+C_{2}=0$, то коэффициент $C_{3}$ не удаётся привести к нулевому значению.

III.В.1. Полагаем, кроме того, что удовлетворяется неравенство $C_{1} \neq 0$. Тогда можно привести к нулевому значению коэффициент $B_{1}$.

III.В.1-1. Дополнительно считаем, что $C_{3} \neq 0, C_{11} \neq 0$. Если $C_{3} \neq 0, C_{11} \neq 0$, то, применяя автоморфизмы А2) и А12) при $\tau$, равном соответственно $\ln \left|C_{1} / C_{3}\right|$ и $-\ln \left|C_{1} / C_{11}\right|$, приводим коэффициенты $C_{1}, C_{3}$ 
и $C_{11}$ к значениям, равным по модулю, и, следовательно, получаем множество простейших представителей вида

$$
\left(\varsigma_{1} \cdot \partial\right)-3\left(\varsigma_{2} \cdot \partial\right) \pm\left(\varsigma_{3} \cdot \partial\right) \pm\left(\varsigma_{11} \cdot \partial\right)+\frac{3}{2}\left(\varsigma_{12} \cdot \partial\right)+D\left(\varsigma_{7} \cdot \partial\right) .
$$

III.B.1-2. Дополнительно принимаем, что выполнены условия $C_{3} \neq 0, C_{11}=0$. Получаем множество простейших представителей

$$
\left(\varsigma_{1} \cdot \partial\right)-3\left(\varsigma_{2} \cdot \partial\right) \pm\left(\varsigma_{3} \cdot \partial\right)+\frac{3}{2}\left(\varsigma_{12} \cdot \partial\right)+D\left(\varsigma_{7} \cdot \partial\right) .
$$

III.В.1-3. Дополнительно принимаем, что выполнены условия $C_{3}=0, C_{11} \neq 0$. $\mathrm{C}$ помощью автоморфизма $\mathrm{A} 12)$ коэффициенты $C_{11}$ и $C_{1}$ удаётся привести к значениям, одинаковым по абсолютной величине. Учитывая ещё, что $3 C_{1}+C_{2}=0$ и $C_{2}=-2 C_{12} \neq 0$, получаем множество простейших представителей

$$
\left(\varsigma_{1} \cdot \partial\right)-3\left(\varsigma_{2} \cdot \partial\right) \pm\left(\varsigma_{11} \cdot \partial\right)+\frac{3}{2}\left(\varsigma_{12} \cdot \partial\right)+D\left(\varsigma_{7} \cdot \partial\right) .
$$

III.В.1-4. Дополнительно принимаем, что выполнены условия $C_{3}=0, C_{11}=0$. Получаем множество простейших представителей

$$
\frac{2}{3}\left(\varsigma_{1} \cdot \partial\right)-2\left(\varsigma_{2} \cdot \partial\right)+D\left(\varsigma_{7} \cdot \partial\right)+\left(\varsigma_{12} \cdot \partial\right) .
$$

III.B.2 Случаи типа III.В.2, когда выполняется равенство $C_{1}=0$, невозможны в рамках предположений III.B.

ЭТАП IV. В пределах этого этапа рассуждений будем считать, что $C_{2}=0$ и $C_{12}=0$. Коэффициенты $C_{10}$ и $C_{11}$ не приводятся к нулю, поэтому придется рассматривать случаи $C_{10} \neq 0, C_{11} \neq 0 ; C_{10} \neq 0, C_{11}=0$; $C_{10}=0, C_{11} \neq 0 ; C_{10}=0, C_{11}=0$. Заметим, что если один из коэффициентов $C_{10}$ или $C_{11}$ равен нулю, то оставшийся из них с помощью автоморфизма А12) приводится к значению \pm 1 .

IVA. Этот случай характеризуется условиями $C_{10} \neq 0, C_{11} \neq 0$. Если $C_{10} \neq 0$ и $C_{11} \neq 0$, то, применяя автоморфизм А12) при $\tau$, равном $\frac{1}{2} \ln \left|C_{11} / C_{10}\right|$, убеждаемся, что $C_{10}$ и $C_{11}$ приводятся к равным по модулю значениям. Рассматриваемый случай удобно разбить еще на два в зависимости от выполнения условий $C_{1} \neq 0$ или $C_{1}=0$.

IV.A.1. Допустим сначала, что $C_{1} \neq 0$. Если $C_{1} \neq 0$, то, применяя автоморфизмы A4) и А3) при $\tau$, соответственно равном $B_{1} / C_{1}$ и $C_{3} /\left(3 C_{1}\right)$, удаётся привести к нулевому значению $B_{1}$ и $C_{3}$. Действуя автоморфизмом А2) при $\tau$, равном $-2 \ln \left|C_{1} / C_{10}\right|$, убеждаемся, что $C_{1}$ и $C_{10}$ приводятся к равным по модулю значениям; применяя затем преобразование умножения, приводим $C_{1}$ к единице и, таким образом, получаем множество простейших представителей следующего вида:

$$
\left(\varsigma_{1} \cdot \partial\right)+D\left(\varsigma_{7} \cdot \partial\right) \pm\left(\varsigma_{10} \cdot \partial\right) \pm\left(\varsigma_{11} \cdot \partial\right) .
$$


IV.A.2. Далее до конца IV.A будем считать, что $C_{1}=0$. Выделим еще восемь вариантов в зависимости от выполнения условий:

IV.A.2-1) $C_{3} \neq 0, B_{1} \neq 0, A_{1} \neq 0$;

IV.A.2-2) $C_{3} \neq 0, B_{1}=0, A_{1} \neq 0$;

IV.A.2-3) $C_{3}=0, B_{1} \neq 0, A_{1} \neq 0$;

IV.A.2-4) $C_{3} \neq 0, B_{1} \neq 0, A_{1}=0$;

IV.A.2-5) $C_{3} \neq 0, B_{1}=0, A_{1}=0$;

IV.A.2-6) $C_{3}=0, B_{1} \neq 0, A_{1}=0$;

IV.A.2-7) $C_{3}=0, B_{1}=0, A_{1} \neq 0$;

IV.A.2-8) $C_{3}=0, B_{1}=0, A_{1}=0$.

IV.A.2-1. Если $C_{3} \neq 0, B_{1} \neq 0, A_{1} \neq 0$, то, применяя автоморфизм A1) при $\tau$, равном $\ln \left|A_{1} / B_{1}\right|$, убеждаемся, что $A_{1}$ и $B_{1}$ приводятся к равным по модулю значениям, и, применяя после этого автоморфизм A2) при $\tau$, равном $\ln \left|A_{1} / C_{3}\right|$, приводим к равным абсолютным значениям величины $A_{1}$ и $C_{3}$. В итоге получаем простейших представителей

$$
\left(\varsigma_{3} \cdot \partial\right) \pm\left(\varsigma_{4} \cdot \partial\right) \pm\left(\varsigma_{7} \cdot \partial\right)+D\left(\left(\varsigma_{10} \cdot \partial\right) \pm\left(\varsigma_{11} \cdot \partial\right)\right) \quad(D \neq 0) .
$$

IV.A.2-2. Считаем выполненными условия $C_{3} \neq 0, B_{1}=0, A_{1} \neq 0$. Применяя автоморфизм А2) при $\tau=-2 \ln \left|A_{1} / C_{10}\right|$, убеждаемся, что $A_{1}$ и $C_{10}$ приводятся к равным по модулю значениям, и, применяя после этого автоморфизм A1) при $\tau=\ln \left|A_{1} / C_{3}\right| / 3$, приводим к равным абсолютным значениям величины $A_{1}$ и $C_{3}$. В итоге получаем простейших представителей

$$
\left(\varsigma_{3} \cdot \partial\right) \pm\left(\varsigma_{7} \cdot \partial\right) \pm\left(\varsigma_{10} \cdot \partial\right) \pm\left(\varsigma_{11} \cdot \partial\right)
$$

IV.A.2-3. Считаем выполненными условия $C_{3}=0, B_{1} \neq 0, A_{1} \neq 0$. Применяя автоморфизм А1) при $\tau=\ln \left|A_{1} / B_{1}\right|$, убеждаемся, что $A_{1}$ и $B_{1}$ приводятся к равным по модулю значениям, и, применяя после этого автоморфизм А2) при $\tau=-2 \ln \left|A_{1} / C_{10}\right|$, приводим к равным абсолютным значениям величины $A_{1}$ и $C_{10}$. В итоге получаем простейших представителей

$$
\left(\varsigma_{4} \cdot \partial\right) \pm\left(\varsigma_{7} \cdot \partial\right) \pm\left(\varsigma_{10} \cdot \partial\right) \pm\left(\varsigma_{11} \cdot \partial\right) .
$$

IV.A.2-4. Считаем выполненными условия $C_{3} \neq 0, B_{1} \neq 0, A_{1}=0$. Применяя автоморфизм A1) при $\tau=\ln \left|1 / B_{1}\right|$, приравниваем абсолютную величину $B_{1}$ к единице, и, применяя после этого автоморфизм A2) при $\tau=\ln \left|1 / C_{3}\right|$, приравниваем $C_{3}=1$. (Применяя автоморфизмы A1) и А2) при $\tau$, связанных равенством $\tau_{(2)}=-2 \tau_{(1)}$, получим преобразование, по своему действию совпадающее с преобразованием умножения, т. е. преобразования A1), А2) и преобразование умножения не являются независимыми.) В итоге получаем простейших представителей

$$
\left(\varsigma_{3} \cdot \partial\right) \pm\left(\varsigma_{4} \cdot \partial\right)+D\left(\left(\varsigma_{10} \cdot \partial\right) \pm\left(\varsigma_{11} \cdot \partial\right)\right) \quad(D \neq 0) .
$$


IV.A.2-5. Считаем выполненными условия $C_{3} \neq 0, B_{1}=0, A_{1}=0$. Применяя автоморфизм $\mathrm{A} 1$ ), находим, что модули коэффициентов $C_{3}$, $C_{10}, C_{11}$ приводятся к одной и той же величине. Поэтому получаем представителей

$$
\left(\varsigma_{3} \cdot \partial\right) \pm\left(\varsigma_{10} \cdot \partial\right) \pm\left(\varsigma_{11} \cdot \partial\right) .
$$

IV.A.2-6. Считаем выполненными условия $C_{3}=0, B_{1} \neq 0, A_{1}=0$. Применяя автоморфизм $\mathrm{A} 1$ ), находим, что модули коэффициентов $B_{1}$, $C_{10}, C_{11}$ приводятся к одной и той же величине. Поэтому получаем представителей

$$
\left(\varsigma_{4} \cdot \partial\right) \pm\left(\varsigma_{10} \cdot \partial\right) \pm\left(\varsigma_{11} \cdot \partial\right) .
$$

IV.A.2-7. Считаем выполненными условия $C_{3}=0, B_{1}=0, A_{1} \neq 0$. Тогда инфинитезимальный оператор группы симметрий будет иметь вид

$$
(\varsigma \cdot \partial)=A_{1}\left(\varsigma_{7} \cdot \partial\right)+C\left(\left(\varsigma_{10} \cdot \partial\right) \pm\left(\varsigma_{11} \cdot \partial\right)\right),
$$

где $|C|=\left|C_{10}\right|=\left|C_{11}\right|$. Следовательно, получаем представителей

$$
\left(\varsigma_{7} \cdot \partial\right)+D\left(\left(\varsigma_{10} \cdot \partial\right) \pm\left(\varsigma_{11} \cdot \partial\right)\right) \quad(D \neq 0) .
$$

IV.A.2-8. Считаем выполненными условия $C_{3}=0, B_{1}=0, A_{1}=0$. Находим простейших представителей

$$
\left(\varsigma_{10} \cdot \partial\right) \pm\left(\varsigma_{11} \cdot \partial\right)
$$

IV.В. Этот случай характеризуется условиями $C_{10} \neq 0, C_{11}=0$.

IV.B.1. Предположим, что $C_{1} \neq 0$. Если $C_{11}=0$, то при $C_{1} \neq 0$ с помощью автоморфизма A12) можно привести к одинаковым абсолютным значениям коэффициенты $C_{10}$ и $C_{1}$; применяя автоморфизм A4) приведем $B_{1}$ к нулевому значению; с помощью автоморфизма А3) к нулю можно привести коэффициент $C_{3}$. Следовательно, получаем простейших представителей

$$
\left(\varsigma_{1} \cdot \partial\right) \pm\left(\varsigma_{10} \cdot \partial\right)+D\left(\varsigma_{7} \cdot \partial\right),
$$

совпадающих с (21) при $D_{1}=0$.

IV.B.2. В случае $C_{1}=0$, действуя далее так же, как и при получении простейших представителей (46)-(53) и учитывая, что $\left|C_{10}\right|$ приводится к единице, если $C_{11}=0$, в каждом из восьми случаев

IV.B.2-1) $C_{3} \neq 0, B_{1} \neq 0, A_{1} \neq 0$;

IV.B.2-2) $C_{3} \neq 0, B_{1}=0, A_{1} \neq 0$;

IV.B.2-3) $C_{3}=0, B_{1} \neq 0, A_{1} \neq 0$;

IV.B.2-4) $C_{3} \neq 0, B_{1} \neq 0, A_{1}=0$

IV.B.2-5) $C_{3} \neq 0, B_{1}=0, A_{1}=0$;

IV.B.2-6) $C_{3}=0, B_{1} \neq 0, A_{1}=0$;

IV.B.2-7) $C_{3}=0, B_{1}=0, A_{1} \neq 0$;

IV.B.2-8) $C_{3}=0, B_{1}=0, A_{1}=0$

получим соответствующих простейших представителей. 
IV.В.2-1. Получаем представителей

$$
\left(\varsigma_{3} \cdot \partial\right) \pm\left(\varsigma_{4} \cdot \partial\right) \pm\left(\varsigma_{7} \cdot \partial\right) \pm\left(\varsigma_{10} \cdot \partial\right) .
$$

IV.В.2-2. Получаем представителей

$$
\left(\varsigma_{3} \cdot \partial\right) \pm\left(\varsigma_{7} \cdot \partial\right) \pm\left(\varsigma_{10} \cdot \partial\right) .
$$

IV.B.2-3. Получаем представителей

$$
\left(\varsigma_{4} \cdot \partial\right) \pm\left(\varsigma_{7} \cdot \partial\right) \pm\left(\varsigma_{10} \cdot \partial\right) .
$$

IV.B.2-4. Получаем представителей

$$
\left(\varsigma_{3} \cdot \partial\right) \pm\left(\varsigma_{4} \cdot \partial\right) \pm\left(\varsigma_{10} \cdot \partial\right) .
$$

IV.B.2-5. Получаем представителей

$$
\left(\varsigma_{3} \cdot \partial\right) \pm\left(\varsigma_{10} \cdot \partial\right) .
$$

IV.B.2-6. Получаем представителей

$$
\left(\varsigma_{4} \cdot \partial\right) \pm\left(\varsigma_{10} \cdot \partial\right) .
$$

IV.B.2-7. Получаем представителей

$$
\left(\varsigma_{7} \cdot \partial\right) \pm\left(\varsigma_{10} \cdot \partial\right) .
$$

IV.B.2-8. Получаем представителя

$$
\left(\varsigma_{10} \cdot \partial\right)
$$

IV.C. Этот случай характеризуется условиями $C_{10}=0, C_{11} \neq 0$.

IV.C.1. Если $C_{10}=0$, то при $C_{1} \neq 0$ приходим к инфинитезимальному оператору

$$
\left(\varsigma_{1} \cdot \partial\right) \pm\left(\varsigma_{11} \cdot \partial\right)+D\left(\varsigma_{7} \cdot \partial\right),
$$

дополняющему (33) при $D_{1}=0$.

IV.C.2. В случае $C_{1}=0$, действуя далее так же, как и при получении простейших представителей (46)-(53), в каждом из восьми случаев

IV.C.2-1) $C_{3} \neq 0, B_{1} \neq 0, A_{1} \neq 0$;

IV.C.2-2) $C_{3} \neq 0, B_{1}=0, A_{1} \neq 0$;

IV.C.2-3) $C_{3}=0, B_{1} \neq 0, A_{1} \neq 0$;

IV.C.2-4) $C_{3} \neq 0, B_{1} \neq 0, A_{1}=0$;

IV.C.2-5) $C_{3} \neq 0, B_{1}=0, A_{1}=0$

IV.C.2-6) $C_{3}=0, B_{1} \neq 0, A_{1}=0$;

IV.C.2-7) $C_{3}=0, B_{1}=0, A_{1} \neq 0$

IV.C.2-8) $C_{3}=0, B_{1}=0, A_{1}=0$

получим соответствующих простейших представителей.

IV.C.2-1. Получаем представителей

$$
\left(\varsigma_{3} \cdot \partial\right) \pm\left(\varsigma_{4} \cdot \partial\right) \pm\left(\varsigma_{7} \cdot \partial\right) \pm\left(\varsigma_{11} \cdot \partial\right) .
$$


IV.C.2-2. Получаем представителей

$$
\left(\varsigma_{3} \cdot \partial\right) \pm\left(\varsigma_{7} \cdot \partial\right) \pm\left(\varsigma_{11} \cdot \partial\right)
$$

IV.C.2-3. Получаем представителей

$$
\left(\varsigma_{4} \cdot \partial\right) \pm\left(\varsigma_{7} \cdot \partial\right) \pm\left(\varsigma_{11} \cdot \partial\right)
$$

IV.C.2-4. Получаем представителей

$$
\left(\varsigma_{3} \cdot \partial\right) \pm\left(\varsigma_{4} \cdot \partial\right) \pm\left(\varsigma_{11} \cdot \partial\right) .
$$

IV.C.2-5. Получаем представителей

$$
\left(\varsigma_{3} \cdot \partial\right) \pm\left(\varsigma_{11} \cdot \partial\right)
$$

IV.C.2-6. Получаем представителей

$$
\left(\varsigma_{4} \cdot \partial\right) \pm\left(\varsigma_{11} \cdot \partial\right)
$$

IV.C.2-7. Получаем представителей

$$
\left(\varsigma_{7} \cdot \partial\right) \pm\left(\varsigma_{11} \cdot \partial\right)
$$

IV.C.2-8. Получаем представителя

$$
\left(\varsigma_{11} \cdot \partial\right)
$$

IV.D. Этот случай характеризуется условиями $C_{10}=0, C_{11}=0$.

IV.D.1. При условии $C_{1} \neq 0$ можно привести к нулю коэффициент $B_{1}$, а с помощью автоморфизма А3) удаётся привести к нулевому значению коэффициент $C_{3}$; в результате приходим к однопараметрическому семейству

$$
\left(\varsigma_{1} \cdot \partial\right)+D\left(\varsigma_{7} \cdot \partial\right) .
$$

IV.D.2. В случае $C_{1}=0$, считая выполненными условия

IV.D.2-1) $C_{3} \neq 0, B_{1} \neq 0, A_{1} \neq 0$;

IV.D.2-2) $C_{3} \neq 0, B_{1}=0, A_{1} \neq 0$;

IV.D.2-3) $C_{3}=0, B_{1} \neq 0, A_{1} \neq 0$;

IV.D.2-4) $C_{3} \neq 0, B_{1} \neq 0, A_{1}=0$;

IV.D.2-5) $C_{3} \neq 0, B_{1}=0, A_{1}=0$;

IV.D.2-6) $C_{3}=0, B_{1} \neq 0, A_{1}=0$;

IV.D.2-7) $C_{3}=0, B_{1}=0, A_{1} \neq 0$,

IV.D.2-8) $C_{3}=0, B_{1}=0, A_{1}=0$

также найдём соответствующих представителей.

IV.D.2-1. Получаем представителей

$$
\left(\varsigma_{3} \cdot \partial\right) \pm\left(\varsigma_{4} \cdot \partial\right) \pm\left(\varsigma_{7} \cdot \partial\right),
$$

которые дополняют (13), (46) при $D=0$. 
IV.D.2-2. Получаем представителей

$$
\left(\varsigma_{3} \cdot \partial\right) \pm\left(\varsigma_{7} \cdot \partial\right)
$$

которые дополняют (14) при $D=0$.

IV.D.2-3. Получаем представителей

$$
\left(\varsigma_{4} \cdot \partial\right) \pm\left(\varsigma_{7} \cdot \partial\right)
$$

которые дополняют (15) при $D=0$.

IV.D.2-4. Получаем представителей

$$
\left(\varsigma_{3} \cdot \partial\right) \pm\left(\varsigma_{4} \cdot \partial\right)
$$

которые дополняют (49) при $D=0$.

IV.D.2-5. Получаем простейшего представителя

$$
\left(\varsigma_{3} \cdot \partial\right)
$$

IV.D.2-6. Получаем простейшего представителя

$$
\left(\varsigma_{4} \cdot \partial\right)
$$

IV.D.2-7. Получаем простейшего представителя

$$
\left(\varsigma_{7} \cdot \partial\right)
$$

который дополняет (19), (52) при $D=0$.

IV.D.2-8. В этом случае получаем нулевой инфинитезимальный оператор.

Перечисленные выше инфинитезимальные операторы образуют оптимальную систему $\Theta_{1}$ одномерных подалгебр естественной конечномерной подалгебры алгебры непрерывных симметрий системы дифференциальных уравнений в частных производных (3). Для удобства восприятия полный список элементов, составляющих оптимальную систему $\Theta_{1}$, приводится в приложении Б, а в приложении В прилагается схема поиска простейших представителей, обеспечивающая наглядное представление реализованного выше алгоритма построения оптимальной системы $\Theta_{1}$.

Построенная оптимальная система одномерных подалгебр естественной конечномерной (размерности 12) подалгебры алгебры симметрий системы дифференциальных уравнений (3) насчитывает один трёхпараметрический элемент, 12 двухпараметрических, 66 однопараметрических элементов и 108 индивидуальных элементов. В этом списке знаки не согласованы и могут быть выбраны независимо. В каждом элементе списка один из базисных операторов $\left(\varsigma_{j} \cdot \partial\right)$ может быть замещен своим коллинеарным аналогом. При построении списка не учтены дискретные симметрии системы дифференциальных уравнений (3).

Заметим, что алгебра симметрий уравнений плоской задачи имеет размерность 7. Оптимальная система одномерных подалгебр состоит из одного двухпараметрического элемента, одиннадцати однопараметрических и двадцати индивидуальных элементов. 
Алгебра симметрий уравнений осесимметричной задачи имеет размерность 5. Оптимальная система одномерных подалгебр состоит из одного однопараметрического элемента и двадцати двух индивидуальных элементов.

Оптимальная система $\Theta_{1}$ используется для редукции системы дифференциальных уравнений в частных производных (3) к системам, содержащим лишь две независимые переменные, которые, в свою очередь, могут быть подвергнуты групповому анализу также с целью их дальнейшей редукции к системам обыкновенных дифференциальных уравнений. Ясно, что этот процесс является достаточно трудоёмким, но при очевидном отсутствии альтернативы единственным имеющимся в распоряжении средством развития теории пространственной задачи теории пластичности.

Свойства симметрии чрезвычайно важны при анализе нелинейных математических моделей, и здесь теоретико-групповые методы (теория групп и алгебр Ли) играют главенствующую роль. В этом плане, несмотря на то, что теория симметрий систем дифференциальных уравнений в частных производных была создана более ста лет тому назад, в математической теории пластичности в настоящее время наблюдается заметный пробел, который должен быть восполнен. Следуя по этому пути, можно будет найти новые точные решения пространственных соотношений теории пластичности. Как свидетельствует проведённый анализ, трёхмерные уравнения Д. Д. Ивлева обладают высокой степенью симметрии, что оставляет надежду получить ряд новых точных решений, описывающих трёхмерное напряжённое состояние идеально пластических тел при условии полной пластичности Хаара-Кармана.

Заключая, заметим, что дифференциальные уравнения математической теории пластичности столь сложны и многообразны, что сейчас трудно рассчитывать на их всеобъемлющее исследование только лишь средствами группового анализа.

Приложение А. Внутренние автоморфизмы алгебры симметрий $L^{12}$

A1) $C_{1}^{\prime}=C_{1}, C_{2}^{\prime}=C_{2}, C_{3}^{\prime}=C_{3} e^{3 \tau}$,

$B_{1}^{\prime}=B_{1} e^{\tau}, B_{2}^{\prime}=B_{2} e^{\tau}, B_{3}^{\prime}=B_{3} e^{\tau}$

$A_{1}^{\prime}=A_{1}, A_{2}^{\prime}=A_{2}, A_{3}^{\prime}=A_{3}$,

$C_{10}^{\prime}=C_{10}, C_{11}^{\prime}=C_{11}, C_{12}^{\prime}=C_{12}$;

A2) $C_{1}^{\prime}=C_{1}, C_{2}^{\prime}=C_{2}, C_{3}^{\prime}=C_{3} e^{\tau}$,

$B_{1}^{\prime}=B_{1}, B_{2}^{\prime}=B_{2}, B_{3}^{\prime}=B_{3}$,

$A_{1}^{\prime}=A_{1}, A_{2}^{\prime}=A_{2}, A_{3}^{\prime}=A_{3}$,

$C_{10}^{\prime}=C_{10} e^{-\tau / 2}, C_{11}^{\prime}=C_{11} e^{-\tau / 2}, C_{12}^{\prime}=C_{12}$;

A3) $C_{1}^{\prime}=C_{1}, C_{2}^{\prime}=C_{2}, C_{3}^{\prime}=C_{3}-3 \tau C_{1}-\tau C_{2}$,

$B_{1}^{\prime}=B_{1}, B_{2}^{\prime}=B_{2}, B_{3}^{\prime}=B_{3}$,

$A_{1}^{\prime}=A_{1}, A_{2}^{\prime}=A_{2}, A_{3}^{\prime}=A_{3}$,

$C_{10}^{\prime}=C_{10}, C_{11}^{\prime}=C_{11}, C_{12}^{\prime}=C_{12}$;

A4) $C_{1}^{\prime}=C_{1}, C_{2}^{\prime}=C_{2}, C_{3}^{\prime}=C_{3}$,

$B_{1}^{\prime}=B_{1}-\tau C_{1}, B_{2}^{\prime}=B_{2}+\tau A_{3}, B_{3}^{\prime}=B_{3}-\tau A_{2}$,

$A_{1}^{\prime}=A_{1}, A_{2}^{\prime}=A_{2}, A_{3}^{\prime}=A_{3}$,

$C_{10}^{\prime}=C_{10}, C_{11}^{\prime}=C_{11}, C_{12}^{\prime}=C_{12}$;

A5) $C_{1}^{\prime}=C_{1}, C_{2}^{\prime}=C_{2}, C_{3}^{\prime}=C_{3}$,

$B_{1}^{\prime}=B_{1}-\tau A_{3}, B_{2}^{\prime}=B_{2}-\tau C_{1}, B_{3}^{\prime}=B_{3}+\tau A_{1}$, 
$A_{1}^{\prime}=A_{1}, A_{2}^{\prime}=A_{2}, A_{3}^{\prime}=A_{3}$,

$C_{10}^{\prime}=C_{10}, C_{11}^{\prime}=C_{11}, C_{12}^{\prime}=C_{12}$;

A6) $C_{1}^{\prime}=C_{1}, C_{2}^{\prime}=C_{2}, C_{3}^{\prime}=C_{3}$,

$B_{1}^{\prime}=B_{1}+\tau A_{2}, B_{2}^{\prime}=B_{2}-\tau A_{1}, B_{3}^{\prime}=B_{3}-\tau C_{1}$,

$A_{1}^{\prime}=A_{1}, A_{2}^{\prime}=A_{2}, A_{3}^{\prime}=A_{3}$,

$C_{10}^{\prime}=C_{10}, C_{11}^{\prime}=C_{11}, C_{12}^{\prime}=C_{12}$

A7) $C_{1}^{\prime}=C_{1}, C_{2}^{\prime}=C_{2}, C_{3}^{\prime}=C_{3}$,

$B_{1}^{\prime}=B_{1}, B_{2}^{\prime}=B_{2} \cos \tau+B_{3} \sin \tau, B_{3}^{\prime}=B_{3} \cos \tau-B_{2} \sin \tau$, $A_{1}^{\prime}=A_{1}, A_{2}^{\prime}=A_{2} \cos \tau+A_{3} \sin \tau, A_{3}^{\prime}=A_{3} \cos \tau-A_{2} \sin \tau$ $C_{10}^{\prime}=C_{10}, C_{11}^{\prime}=C_{11}, C_{12}^{\prime}=C_{12}$;

A8) $C_{1}^{\prime}=C_{1}, C_{2}^{\prime}=C_{2}, C_{3}^{\prime}=C_{3}$,

$B_{1}^{\prime}=B_{1} \cos \tau-B_{3} \sin \tau, B_{2}^{\prime}=B_{2}, B_{3}^{\prime}=B_{3} \cos \tau+B_{1} \sin \tau$ $A_{1}^{\prime}=A_{1} \cos \tau-A_{3} \sin \tau, A_{2}^{\prime}=A_{2}, A_{3}^{\prime}=A_{3} \cos \tau+A_{1} \sin \tau$ $C_{10}^{\prime}=C_{10}, C_{11}^{\prime}=C_{11}, C_{12}^{\prime}=C_{12}$;

A9) $C_{1}^{\prime}=C_{1}, C_{2}^{\prime}=C_{2}, C_{3}^{\prime}=C_{3}$,

$B_{1}^{\prime}=B_{1} \cos \tau+B_{2} \sin \tau, B_{2}^{\prime}=B_{2} \cos \tau-B_{1} \sin \tau, B_{3}^{\prime}=B_{3}$,

$A_{1}^{\prime}=A_{1} \cos \tau+A_{2} \sin \tau, A_{2}^{\prime}=A_{2} \cos \tau-A_{1} \sin \tau, A_{3}^{\prime}=A_{3}$,

$C_{10}^{\prime}=C_{10}, C_{11}^{\prime}=C_{11}, C_{12}^{\prime}=C_{12}$;

A10) $C_{1}^{\prime}=C_{1}, C_{2}^{\prime}=C_{2}, C_{3}^{\prime}=C_{3}$,

$B_{1}^{\prime}=B_{1}, B_{2}^{\prime}=B_{2}, B_{3}^{\prime}=B_{3}$,

$A_{1}^{\prime}=A_{1}, A_{2}^{\prime}=A_{2}, A_{3}^{\prime}=A_{3}$,

$C_{10}^{\prime}=C_{10}+\frac{1}{2} \tau C_{2}-\tau C_{12}, C_{11}^{\prime}=C_{11}, C_{12}^{\prime}=C_{12}$;

A11) $C_{1}^{\prime}=C_{1}, C_{2}^{\prime}=C_{2}, C_{3}^{\prime}=C_{3}$,

$B_{1}^{\prime}=B_{1}, B_{2}^{\prime}=B_{2}, B_{3}^{\prime}=B_{3}$,

$A_{1}^{\prime}=A_{1}, A_{2}^{\prime}=A_{2}, A_{3}^{\prime}=A_{3}$,

$C_{10}^{\prime}=C_{10}, C_{11}^{\prime}=C_{11}+\frac{1}{2} \tau C_{2}+\tau C_{12}, C_{12}^{\prime}=C_{12}$;

A12) $C_{1}^{\prime}=C_{1}, C_{2}^{\prime}=C_{2}, C_{3}^{\prime}=C_{3}$,

$B_{1}^{\prime}=B_{1}, B_{2}^{\prime}=B_{2}, B_{3}^{\prime}=B_{3}$

$A_{1}^{\prime}=A_{1}, A_{2}^{\prime}=A_{2}, A_{3}^{\prime}=A_{3}$,

$C_{10}^{\prime}=C_{10} e^{\tau}, C_{11}^{\prime}=C_{11} e^{-\tau}, C_{12}^{\prime}=C_{12}$.

\section{Приложение Б. Оптимальная система $\Theta_{1}$ алгебры Ли $L^{12}$}

Б1) $\left(\varsigma_{1} \cdot \partial\right)+D_{1}\left(\varsigma_{2} \cdot \partial\right)+D_{2}\left(\varsigma_{7} \cdot \partial\right)+D_{3}\left(\varsigma_{12} \cdot \partial\right),\left|D_{1}\right| \neq 2\left|D_{3}\right|$

Б2) $\left(\varsigma_{2} \cdot \partial\right) \pm\left(\varsigma_{4} \cdot \partial\right)+D_{1}\left(\varsigma_{7} \cdot \partial\right)+D_{2}\left(\varsigma_{12} \cdot \partial\right)$;

Б3) $\left(\varsigma_{2} \cdot \partial\right)+D_{1}\left(\varsigma_{7} \cdot \partial\right)+D_{2}\left(\varsigma_{12} \cdot \partial\right)$;

Б4) $\left(\varsigma_{1} \cdot \partial\right)-3\left(\varsigma_{2} \cdot \partial\right) \pm\left(\varsigma_{3} \cdot \partial\right)+D_{1}\left(\varsigma_{7} \cdot \partial\right)+D_{2}\left(\varsigma_{12} \cdot \partial\right)$;

Б5) $\left(\varsigma_{1} \cdot \partial\right)-3\left(\varsigma_{2} \cdot \partial\right)+D_{1}\left(\varsigma_{7} \cdot \partial\right)+D_{2}\left(\varsigma_{12} \cdot \partial\right)$;

Б6) $\left(\varsigma_{3} \cdot \partial\right) \pm\left(\varsigma_{4} \cdot \partial\right) \pm\left(\varsigma_{7} \cdot \partial\right)+D\left(\varsigma_{12} \cdot \partial\right), D \neq 0$;

Б7) $\left(\varsigma_{3} \cdot \partial\right) \pm\left(\varsigma_{7} \cdot \partial\right)+D\left(\varsigma_{12} \cdot \partial\right), D \neq 0$;

Б8) $\left(\varsigma_{4} \cdot \partial\right) \pm\left(\varsigma_{7} \cdot \partial\right)+D\left(\varsigma_{12} \cdot \partial\right), D \neq 0$;

Б9) $\left(\varsigma_{3} \cdot \partial\right) \pm\left(\varsigma_{4} \cdot \partial\right) \pm\left(\varsigma_{12} \cdot \partial\right)$;

Б10) $\left(\varsigma_{3} \cdot \partial\right) \pm\left(\varsigma_{12} \cdot \partial\right)$;

Б11) $\left(\varsigma_{4} \cdot \partial\right) \pm\left(\varsigma_{12} \cdot \partial\right)$; 
Б12) $\left(\varsigma_{7} \cdot \partial\right)+D\left(\varsigma_{12} \cdot \partial\right), D \neq 0$

Б13) $\left(\varsigma_{12} \cdot \partial\right)$;

Б14) $\left(\varsigma_{1} \cdot \partial\right) \pm\left(\varsigma_{10} \cdot \partial\right)+D_{1}\left(\left(\varsigma_{2} \cdot \partial\right)+\frac{1}{2}\left(\varsigma_{12} \cdot \partial\right)\right)+D_{2}\left(\varsigma_{7} \cdot \partial\right), D_{1} \neq 0$;

Б15) $\left(\varsigma_{1} \cdot \partial\right)+D_{1}\left(\left(\varsigma_{2} \cdot \partial\right)+\frac{1}{2}\left(\varsigma_{12} \cdot \partial\right)\right)+D_{2}\left(\varsigma_{7} \cdot \partial\right), D_{1} \neq 0$, дополняет Б1) при $D_{3}=D_{1} / 2$;

Б16) $\left(\varsigma_{2} \cdot \partial\right) \pm\left(\varsigma_{4} \cdot \partial\right) \pm\left(\varsigma_{10} \cdot \partial\right)+\frac{1}{2}\left(\varsigma_{12} \cdot \partial\right)+D\left(\varsigma_{7} \cdot \partial\right)$;

Б17) $\left(\varsigma_{2} \cdot \partial\right) \pm\left(\varsigma_{10} \cdot \partial\right)+\frac{1}{2}\left(\varsigma_{12} \cdot \partial\right)+D\left(\varsigma_{7} \cdot \partial\right)$;

Б18) $2\left(\varsigma_{2} \cdot \partial\right)+D\left(\left(\varsigma_{4} \cdot \partial\right) \pm\left(\varsigma_{7} \cdot \partial\right)\right)+\left(\varsigma_{12} \cdot \partial\right), D \neq 0$;

Б19) $2\left(\varsigma_{2} \cdot \partial\right) \pm\left(\varsigma_{4} \cdot \partial\right)+\left(\varsigma_{12} \cdot \partial\right)$;

Б20) $2\left(\varsigma_{2} \cdot \partial\right)+D\left(\varsigma_{7} \cdot \partial\right)+\left(\varsigma_{12} \cdot \partial\right), D \neq 0$;

Б21) $2\left(\varsigma_{2} \cdot \partial\right)+\left(\varsigma_{12} \cdot \partial\right)$, дополняет Б18), Б20) при $D=0$;

Б22) $\left(\varsigma_{1} \cdot \partial\right)-3\left(\varsigma_{2} \cdot \partial\right) \pm\left(\varsigma_{3} \cdot \partial\right) \pm\left(\varsigma_{10} \cdot \partial\right)-\frac{3}{2}\left(\varsigma_{12} \cdot \partial\right)+D\left(\varsigma_{7} \cdot \partial\right)$;

Б23) $\left(\varsigma_{1} \cdot \partial\right)-3\left(\varsigma_{2} \cdot \partial\right) \pm\left(\varsigma_{3} \cdot \partial\right)-\frac{3}{2}\left(\varsigma_{12} \cdot \partial\right)+D\left(\varsigma_{7} \cdot \partial\right)$;

Б24) $\left(\varsigma_{1} \cdot \partial\right)-3\left(\varsigma_{2} \cdot \partial\right) \pm\left(\varsigma_{10} \cdot \partial\right)-\frac{3}{2}\left(\varsigma_{12} \cdot \partial\right)+D\left(\varsigma_{7} \cdot \partial\right)$;

Б25) $-\frac{2}{3}\left(\varsigma_{1} \cdot \partial\right)+2\left(\varsigma_{2} \cdot \partial\right)+D\left(\varsigma_{7} \cdot \partial\right)+\left(\varsigma_{12} \cdot \partial\right)$;

Б26) $\left(\varsigma_{1} \cdot \partial\right) \pm\left(\varsigma_{11} \cdot \partial\right)+D_{1}\left(\left(\varsigma_{2} \cdot \partial\right)-\frac{1}{2}\left(\varsigma_{12} \cdot \partial\right)\right)+D_{2}\left(\varsigma_{7} \cdot \partial\right), D_{1} \neq 0$;

Б27) $\left(\varsigma_{1} \cdot \partial\right)+D_{1}\left(\left(\varsigma_{2} \cdot \partial\right)-\frac{1}{2}\left(\varsigma_{12} \cdot \partial\right)\right)+D_{2}\left(\varsigma_{7} \cdot \partial\right), D_{1} \neq 0$, дополняет Б1) при $D_{3}=-D_{1} / 2$;

Б28) $\left(\varsigma_{2} \cdot \partial\right) \pm\left(\varsigma_{4} \cdot \partial\right) \pm\left(\varsigma_{11} \cdot \partial\right)-\frac{1}{2}\left(\varsigma_{12} \cdot \partial\right)+D\left(\varsigma_{7} \cdot \partial\right)$;

Б29) $\left(\varsigma_{2} \cdot \partial\right) \pm\left(\varsigma_{11} \cdot \partial\right)-\frac{1}{2}\left(\varsigma_{12} \cdot \partial\right)+D\left(\varsigma_{7} \cdot \partial\right)$;

Б30) $-2\left(\varsigma_{2} \cdot \partial\right)+D\left(\left(\varsigma_{4} \cdot \partial\right) \pm\left(\varsigma_{7} \cdot \partial\right)\right)+\left(\varsigma_{12} \cdot \partial\right), D \neq 0$;

Б31) $-2\left(\varsigma_{2} \cdot \partial\right) \pm\left(\varsigma_{4} \cdot \partial\right)+\left(\varsigma_{12} \cdot \partial\right)$;

Б32) $-2\left(\varsigma_{2} \cdot \partial\right)+D\left(\varsigma_{7} \cdot \partial\right)+\left(\varsigma_{12} \cdot \partial\right), D \neq 0$;

Б33) $-2\left(\varsigma_{2} \cdot \partial\right)+\left(\varsigma_{12} \cdot \partial\right)$, дополняет Б30), Б32) при $D=0$;

Б34) $\left(\varsigma_{1} \cdot \partial\right)-3\left(\varsigma_{2} \cdot \partial\right) \pm\left(\varsigma_{3} \cdot \partial\right) \pm\left(\varsigma_{11} \cdot \partial\right)+\frac{3}{2}\left(\varsigma_{12} \cdot \partial\right)+D\left(\varsigma_{7} \cdot \partial\right)$;

Б35) $\left(\varsigma_{1} \cdot \partial\right)-3\left(\varsigma_{2} \cdot \partial\right) \pm\left(\varsigma_{3} \cdot \partial\right)+\frac{3}{2}\left(\varsigma_{12} \cdot \partial\right)+D\left(\varsigma_{7} \cdot \partial\right)$;

Б36) $\left(\varsigma_{1} \cdot \partial\right)-3\left(\varsigma_{2} \cdot \partial\right) \pm\left(\varsigma_{11} \cdot \partial\right)+\frac{3}{2}\left(\varsigma_{12} \cdot \partial\right)+D\left(\varsigma_{7} \cdot \partial\right)$;

Б37) $\frac{2}{3}\left(\varsigma_{1} \cdot \partial\right)-2\left(\varsigma_{2} \cdot \partial\right)+D\left(\varsigma_{7} \cdot \partial\right)+\left(\varsigma_{12} \cdot \partial\right)$;

Б38) $\left(\varsigma_{1} \cdot \partial\right)+D\left(\varsigma_{7} \cdot \partial\right) \pm\left(\varsigma_{10} \cdot \partial\right) \pm\left(\varsigma_{11} \cdot \partial\right)$

Б39) $\left(\varsigma_{3} \cdot \partial\right) \pm\left(\varsigma_{4} \cdot \partial\right) \pm\left(\varsigma_{7} \cdot \partial\right)+D\left(\left(\varsigma_{10} \cdot \partial\right) \pm\left(\varsigma_{11} \cdot \partial\right)\right), D \neq 0$;

Б40) $\left(\varsigma_{3} \cdot \partial\right) \pm\left(\varsigma_{7} \cdot \partial\right) \pm\left(\varsigma_{10} \cdot \partial\right) \pm\left(\varsigma_{11} \cdot \partial\right)$;

Б41) $\left(\varsigma_{4} \cdot \partial\right) \pm\left(\varsigma_{7} \cdot \partial\right) \pm\left(\varsigma_{10} \cdot \partial\right) \pm\left(\varsigma_{11} \cdot \partial\right)$;

Б42) $\left(\varsigma_{3} \cdot \partial\right) \pm\left(\varsigma_{4} \cdot \partial\right)+D\left(\left(\varsigma_{10} \cdot \partial\right) \pm\left(\varsigma_{11} \cdot \partial\right)\right), D \neq 0$;

Б43) $\left(\varsigma_{3} \cdot \partial\right) \pm\left(\varsigma_{10} \cdot \partial\right) \pm\left(\varsigma_{11} \cdot \partial\right)$;

Б44) $\left(\varsigma_{4} \cdot \partial\right) \pm\left(\varsigma_{10} \cdot \partial\right) \pm\left(\varsigma_{11} \cdot \partial\right)$;

Б45) $\left(\varsigma_{7} \cdot \partial\right)+D\left(\left(\varsigma_{10} \cdot \partial\right) \pm\left(\varsigma_{11} \cdot \partial\right)\right), D \neq 0$;

Б46) $\left(\varsigma_{10} \cdot \partial\right) \pm\left(\varsigma_{11} \cdot \partial\right)$;

Б47) $\left(\varsigma_{1} \cdot \partial\right) \pm\left(\varsigma_{10} \cdot \partial\right)+D\left(\varsigma_{7} \cdot \partial\right)$, дополняет Б14) при $D_{1}=0$;

Б48) $\left(\varsigma_{3} \cdot \partial\right) \pm\left(\varsigma_{4} \cdot \partial\right) \pm\left(\varsigma_{7} \cdot \partial\right) \pm\left(\varsigma_{10} \cdot \partial\right)$;

Б49) $\left(\varsigma_{3} \cdot \partial\right) \pm\left(\varsigma_{7} \cdot \partial\right) \pm\left(\varsigma_{10} \cdot \partial\right)$;

Б50) $\left(\varsigma_{4} \cdot \partial\right) \pm\left(\varsigma_{7} \cdot \partial\right) \pm\left(\varsigma_{10} \cdot \partial\right)$;

Б51) $\left(\varsigma_{3} \cdot \partial\right) \pm\left(\varsigma_{4} \cdot \partial\right) \pm\left(\varsigma_{10} \cdot \partial\right)$;

Б52) $\left(\varsigma_{3} \cdot \partial\right) \pm\left(\varsigma_{10} \cdot \partial\right)$;

Б53) $\left(\varsigma_{4} \cdot \partial\right) \pm\left(\varsigma_{10} \cdot \partial\right)$; 


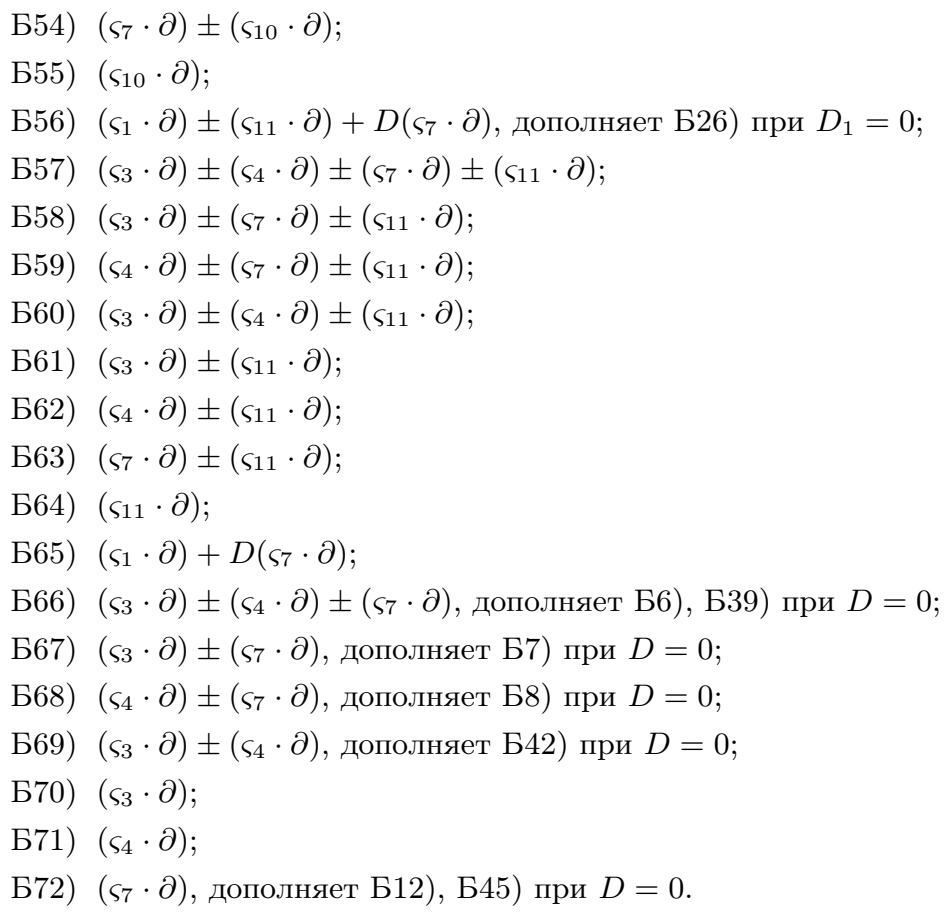

Приложение $\mathrm{B}^{1}$. Алгоритм построения оптимальной системы $\Theta_{1}$ алгебры Ли $L^{12}$

Б1-613: $C_{2} \pm 2 C_{12} \neq 0$,

Б1-63: $3 C_{1}+C_{2} \neq 0$,

Б1: $C_{1} \neq 0$;

Б2: $C_{1}=0, C_{2} \neq 0, B_{1} \neq 0$;

Б3: $C_{1}=0, C_{2} \neq 0, B_{1}=0$;

Б4-Б13: $3 C_{1}+C_{2}=0$,

Б4: $C_{1} \neq 0, C_{3} \neq 0$;

Б5: $C_{1} \neq 0, C_{3}=0$;

Б6: $C_{1}=0, C_{3} \neq 0, B_{1} \neq 0, A_{1} \neq 0$;

Б7: $C_{1}=0, C_{3} \neq 0, B_{1}=0, A_{1} \neq 0$;

Б8: $C_{1}=0, C_{3}=0, B_{1} \neq 0, A_{1} \neq 0$;

Б9: $C_{1}=0, C_{3} \neq 0, B_{1} \neq 0, A_{1}=0$;

Б10: $C_{1}=0, C_{3} \neq 0, B_{1}=0, A_{1}=0$;

Б11: $C_{1}=0, C_{3}=0, B_{1} \neq 0, A_{1}=0$;

Б12: $C_{1}=0, C_{3}=0, B_{1}=0, A_{1} \neq 0$;

Б13: $C_{1}=0, C_{3}=0, B_{1}=0, A_{1}=0$;

Б14-625: $C_{2}-2 C_{12}=0, C_{2}+2 C_{12} \neq 0$,

Б14-Б21: $3 C_{1}+C_{2} \neq 0$,

Б14: $C_{1} \neq 0, C_{10} \neq 0$;

Б15: $C_{1} \neq 0, C_{10}=0$;

Б16: $C_{1}=0, C_{10} \neq 0, B_{1} \neq 0, C_{2} \neq 0$

Б17: $C_{1}=0, C_{10} \neq 0, B_{1}=0, C_{2} \neq 0$;

Б18: $C_{1}=0, C_{10}=0, B_{1} \neq 0, A_{1} \neq 0, C_{2} \neq 0$;

Б19: $C_{1}=0, C_{10}=0, B_{1} \neq 0, A_{1}=0, C_{2} \neq 0$;

Б20: $C_{1}=0, C_{10}=0, B_{1}=0, A_{1} \neq 0, C_{2} \neq 0$;

${ }^{1}$ Номера соответствуют строкам в списке элементов оптимальной системы $\Theta_{1}$ приложения Б. 
Б21: $C_{1}=0, C_{10}=0, B_{1}=0, A_{1}=0, C_{2} \neq 0$;

622-625: $3 C_{1}+C_{2}=0$

Б22: $C_{1} \neq 0, C_{3} \neq 0, C_{10} \neq 0$;

Б23: $C_{1} \neq 0, C_{3} \neq 0, C_{10}=0$;

Б24: $C_{1} \neq 0, C_{3}=0, C_{10} \neq 0$;

Б25: $C_{1} \neq 0, C_{3}=0, C_{10}=0$;

Б26-Б37: $C_{2}+2 C_{12}=0, C_{2}-2 C_{12} \neq 0$,

Б26-Б33: $3 C_{1}+C_{2} \neq 0$

Б26: $C_{1} \neq 0, C_{11} \neq 0$;

Б27: $C_{1} \neq 0, C_{11}=0$;

Б28: $C_{1}=0, C_{11} \neq 0, B_{1} \neq 0, C_{2} \neq 0$;

Б29: $C_{1}=0, C_{11} \neq 0, B_{1}=0, C_{2} \neq 0$;

Б30: $C_{1}=0, C_{11}=0, B_{1} \neq 0, A_{1} \neq 0, C_{2} \neq 0$;

Б31: $C_{1}=0, C_{11}=0, B_{1} \neq 0, A_{1}=0, C_{2} \neq 0$;

Б32: $C_{1}=0, C_{11}=0, B_{1}=0, A_{1} \neq 0, C_{2} \neq 0$;

Б33: $C_{1}=0, C_{11}=0, B_{1}=0, A_{1}=0, C_{2} \neq 0$;

Б34-637: $3 C_{1}+C_{2}=0$,

Б34: $C_{1} \neq 0, C_{3} \neq 0, C_{11} \neq 0$;

Б35: $C_{1} \neq 0, C_{3} \neq 0, C_{11}=0$;

Б36: $C_{1} \neq 0, C_{3}=0, C_{11} \neq 0$;

Б37: $C_{1} \neq 0, C_{3}=0, C_{11}=0$;

Б38-672: $C_{2}=0, C_{12}=0$,

Б38-Б46: $C_{10} \neq 0, C_{11} \neq 0$,

Б38: $C_{1} \neq 0$

Б39: $C_{1}=0, C_{3} \neq 0, B_{1} \neq 0, A_{1} \neq 0$;

Б40: $C_{1}=0, C_{3} \neq 0, B_{1}=0, A_{1} \neq 0$;

Б41: $C_{1}=0, C_{3}=0, B_{1} \neq 0, A_{1} \neq 0$;

Б42: $C_{1}=0, C_{3} \neq 0, B_{1} \neq 0, A_{1}=0$;

Б43: $C_{1}=0, C_{3} \neq 0, B_{1}=0, A_{1}=0$;

Б44: $C_{1}=0, C_{3}=0, B_{1} \neq 0, A_{1}=0$;

Б45: $C_{1}=0, C_{3}=0, B_{1}=0, A_{1} \neq 0$;

Б46: $C_{1}=0, C_{3}=0, B_{1}=0, A_{1}=0$;

Б47-655: $C_{10} \neq 0, C_{11}=0$,

Б47: $C_{1} \neq 0$;

Б48: $C_{1}=0, C_{3} \neq 0, B_{1} \neq 0, A_{1} \neq 0$;

Б49: $C_{1}=0, C_{3} \neq 0, B_{1}=0, A_{1} \neq 0$;

Б50: $C_{1}=0, C_{3}=0, B_{1} \neq 0, A_{1} \neq 0$;

Б51: $C_{1}=0, C_{3} \neq 0, B_{1} \neq 0, A_{1}=0$;

Б52: $C_{1}=0, C_{3} \neq 0, B_{1}=0, A_{1}=0$;

Б53: $C_{1}=0, C_{3}=0, B_{1} \neq 0, A_{1}=0$;

554: $C_{1}=0, C_{3}=0, B_{1}=0, A_{1} \neq 0$;

Б55: $C_{1}=0, C_{3}=0, B_{1}=0, A_{1}=0$;

Б56-Б64: $C_{10}=0, C_{11} \neq 0$,

Б56: $C_{1} \neq 0$;

Б57: $C_{1}=0, C_{3} \neq 0, B_{1} \neq 0, A_{1} \neq 0$;

Б58: $C_{1}=0, C_{3} \neq 0, B_{1}=0, A_{1} \neq 0$;

Б59: $C_{1}=0, C_{3}=0, B_{1} \neq 0, A_{1} \neq 0$;

Б60: $C_{1}=0, C_{3} \neq 0, B_{1} \neq 0, A_{1}=0$;

Б61: $C_{1}=0, C_{3} \neq 0, B_{1}=0, A_{1}=0$;

Б62: $C_{1}=0, C_{3}=0, B_{1} \neq 0, A_{1}=0$;

Б63: $C_{1}=0, C_{3}=0, B_{1}=0, A_{1} \neq 0$;

Б64: $C_{1}=0, C_{3}=0, B_{1}=0, A_{1}=0$;

Б65-672: $C_{10}=0, C_{11}=0$,

Б65: $C_{1} \neq 0$; 
Б66: $C_{1}=0, C_{3} \neq 0, B_{1} \neq 0, A_{1} \neq 0$;

Б67: $C_{1}=0, C_{3} \neq 0, B_{1}=0, A_{1} \neq 0$;

Б68: $C_{1}=0, C_{3}=0, B_{1} \neq 0, A_{1} \neq 0$;

Б69: $C_{1}=0, C_{3} \neq 0, B_{1} \neq 0, A_{1}=0$;

Б70: $C_{1}=0, C_{3} \neq 0, B_{1}=0, A_{1}=0$;

Б71: $C_{1}=0, C_{3}=0, B_{1} \neq 0, A_{1}=0$;

Б72: $C_{1}=0, C_{3}=0, B_{1}=0, A_{1} \neq 0$.

\section{БИБЛИОГРАФИЧЕСКИЙ СПИСОК}

1. Hill R. The Mathematical Theory of Plasticity: Reprint of the 1950 original. Vol. 11 / Oxford Classic Texts in the Physical Sciences. Oxford Engineering Science Series. New York: The Clarendon Press, Oxford University Press, 1988. 366 рр.; русск. пер.: Хилл Р. Математическая теория пластичности. М.: Гостехтеоретиздат, 1956. 407 с.

2. Freudental A.M., Geiringer H. The mathematical theories of the inelastic continuum / In: Handbuch der Physik. Vol. 6: Elastizität und Plastizität; ed. S. Flügge. Berlin - Göttingen - Heidelberg: Springer-Verlag, 1958. Pp. 229-433; русск. пер.: Фрейденталь А., Гейрингер X. Математические теории неупругой сплошной среды. М.: Физматгиз, 1962. 432 с.

3. Качанов Л. М. Основы теории пластичности. М.: Наука, 1969. 420 с. [Kachanov L. M. Principles of Plasticity Theory. Moscow: Nauka, 1969. 420 pp.]

4. Соколовский В. В. Теория пластичности. М.: Высш. шк., 1969. 608 с. [Sokolovsky V. V. Theory of plasticity. Moscow: Vyssh. shk., 1969. 608 pp.]

5. Ивлев Д. Д. Теория идеальной пластичности. М.: Наука, 1966. 232 с. [Ivlev D. D. Theory of ideal plasticity. Moscow: Nauka, 1966. 232 pp.]

6. Ивлев Д. Д. Мир эллиптический и Мир гиперболический // Вестн. Самар. гос. унив. Ecтественнонаучн. сер., 2005. № 5(39). C. 33-41. [Ivlev D. D. The world is elliptic and the world is hyperbolic// Vestn. Samar. Gos. Univ. Estestvennonauchn. Ser., 2005. no. 5(39). Pp. 33-41].

7. Ивлев Д.Д. О соотношениях, определяющих пластическое течение при условии пластичности Треска, и его обобщениях // Докл. АН CCCP, 1959. Т. 124, № 3. С. 546-549; англ. пер.: Ivlev D. D. On relations defining plastic flow under Tresca condition of plasticity and its generalizations // Sov. Phys. Dokl., 1959. Vol.4. Pp. 217-220.

8. Радаев Ю.Н. О канонических преобразованиях Пуанкаре и инвариантах уравнений пластического равновесия // Изв. АН СCCP. MTT, 1990. №1. C. 86-94. [Radaev Yu. N. On Poincaré's canonical transformations and the invariants of the plastic equilibrium equations // Izv. AN SSSR. MTT, 1990. no. 1. Pp. 86-94].

9. Радаев Ю. Н. К теории трехмерных уравнений математической теории пластичности // Изв. РАН. МTT, 2003. № 5. С. 102-120. [Radaev Yu. N. On the theory of three-dimensional equations of the mathematical theory of plasticity // Izv. RAN. MTT, 2003. no. 5. Pp. 102120].

10. Радаев Ю. Н. Пространственная задача математической теории пластичности. Самара: Изд-во Самар. унив., 2006. 340 с. [Radaev Yu. N. Spatial Problem of Mathematical Theory of Plasticity. Samara: Izd-vo Samar. Univ., 2006. 340 pp.]

11. Овсянников Л. В. Групповой анализ дифференциальных уравнений. М.: Наука, 1978. 399 c. [Ovsjannikov L. V. Group analysis of differential equations. Moscow: Nauka, 1978. 399 pp.]

12. Olver P. J. Application of Lie Groups to Differential Equations. Vol. 107 / Graduate Texts in Mathematics. New York: Springer, 1986. 497 pp.; русск. пер.: Олвер П. Приложения групп Ли к дифференциальным уравнениям. М.: Мир, 1989. 639 с.

13. Olver P.J. Equivalence, Invariants, and Symmetry. Cambridge, New York, Melbourne: Cambridge University Press, 1995. 526 pp.

14. Ковалев В.А., Радаев Ю.Н. Элементы теории поля: вариационные симметрии и геометрические инварианты. М.: Физматлит, 2009. 156 с. [Kovalev V.A., Radaev Yu.N. 
Elements of the classical field theory: variational symmetries and geometric invariants. Moscow: Fizmatlit, 2009. 156 pp.]

\section{AN OPTIMAL SYSTEM OF ONE-DIMENSIONAL SUBALGEBRAS FOR THE SYMMETRY ALGEBRA OF THREE-DIMENSIONAL EQUATIONS OF THE PERFECT PLASTICITY}

\section{V.A. Kovalev ${ }^{1}$, Yu. N. Radaev ${ }^{2}$}

1 Moscow City Government University of Management Moscow, 28, Sretenka st., Moscow, 107045, Russia.

2 A. Ishlinsky Institite for Problems in Mechanics, Russian Academy of Sciences, 101, pr. Vernadskogo, Moscow, 119526, Russia.

E-mail: vlad_koval@mail.ru; y.radayev@gmail.com

The present paper is devoted to a study of a natural 12-dimensional symmetry algebra of the three-dimensional hyperbolic differential equations of the perfect plasticity, obtained by D. D. Ivlev in 1959 and formulated in isostatic coordinates. An optimal system of one-dimensional subalgebras constructing algorithm for the Lie algebra is proposed. The optimal system (total 187 elements) is shown consist of a 3-parametrical element, twelve 2-parametrical elements, sixty six 1-parametrical elements and one hundred and eight individual elements.

Key words: theory of plasticity, isostatic coordinate, symmetry group, symmetry algebra, subalgebra, optimal system, algorithm.

Original article submitted 20/XII/2010; revision submitted $18 / \mathrm{II} / 2011$.

Vladimir A. Kovalev (Dr. Sc. (Phys. \& Math.)), Head of Dept., Dept. of Applied Mathematics. Yuriy N. Radaev (Dr. Sc. (Phys. \& Math.)), Leading Researcher, Lab. of Modeling in Solid Mechanics. 